\title{
Light-cone distribution amplitudes of the nucleon and
} $\Delta$ baryon

\author{
June-Young Kim, ${ }^{a}$ Hyun-Chul Kim ${ }^{b, c}$ and Maxim V. Polyakov ${ }^{a, d, 1}$ \\ ${ }^{a}$ Institut für Theoretische Physik II, Fakultät für Physik und Astronomie, Ruhr-Universität Bochum, \\ Universitätsstraße 150, D-44780 Bochum, Germany \\ ${ }^{b}$ Department of Physics, Inha University, \\ 100 Inha-ro, Michuhol-gu, Incheon 22212, Republic of Korea \\ ${ }^{c}$ School of Physics, Korea Institute for Advanced Study (KIAS), \\ 85 Hoegiro, Dongdaemun-gu, Seoul 02455, Republic of Korea \\ ${ }^{d}$ Petersburg Nuclear Physics Institute, \\ Gatchina, 188300, St. Petersburg, Russia \\ E-mail: Jun-Young.Kim@ruhr-uni-bochum.de, hchkim@inha.ac.kr
}

AbStract: We investigate the light-cone wave functions and leading-twist distribution amplitudes for the nucleon and $\Delta$ baryon within the framework of the chiral quark-soliton model. The baryon wave function consists of the valence quark and vacuum wave functions. The vacuum wave functions generate all possible higher Fock states by expanding them. We find that it is essential to consider the five-quark component and relativistic corrections to evaluate the distribution amplitudes of the nucleon and $\Delta$ isobar. Having taken into account them, we derive the distribution amplitudes. The results are in good agreement with the lattice data.

KEYwords: Phenomenological Models, QCD Phenomenology

ARXIV EPRINT: 2110.05889

On August 25, 2021 our respected and beloved colleague and friend Maxim Vladimirovich Polyakov passed away. The present work has been done under his coaching. We dedicate it to the memory of our friend Maxim Vladimirovich Polyakov.

\footnotetext{
${ }^{1}$ Deceased.
} 


\section{Contents}

1 Introduction 1

2 Nucleon distribution amplitudes $\quad 3$

$3 \quad \Delta$ baryon distribution amplitudes $\quad 5$

4 Light-cone wave function in the chiral quark-soliton model 6

$\begin{array}{lll}4.1 & \text { Baryon wave functions } & 7\end{array}$

4.2 Quantization 8

4.3 Baryon light-cone wave functions 9

4.4 Fock components of the baryon light-cone wave functions 12

$\begin{array}{lll}4.4 .1 & 3 Q \text { Fock component } & 12\end{array}$

$\begin{array}{lll}\text { 4.4.2 } 5 Q \text { Fock component } & 13\end{array}$

$\begin{array}{lll}4.5 & \text { Distribution amplitudes } & 15\end{array}$

5 Numerical results and discussion $\quad 16$

$\begin{array}{llr}6 & \text { Summary and conclusions } & 20\end{array}$

$\begin{array}{ll}\text { A Baryon rotational wave functions } & 21\end{array}$

$\begin{array}{ll}\text { B Distribution amplitudes } & 23\end{array}$

\section{Introduction}

Understanding the quark-gluon structure of the nucleon has been one of the most important issues in hadronic physics. The nucleon light-cone wave function (LCWF) provides a systematic way of examining it, since it is decomposed unequivocally by the Fock states of quark-gluon degrees of freedom on the light cone [1-3]. This LCWF is derived by projecting the nucleon state on the Fock basis that consists of various partons, i.e., quark $(Q)$, antiquark $(\bar{Q})$ and gluon $(g)$. The LCWF is considered as a basic building block in high-energy reactions. The $3 Q$ Fock component of the LCWF arises as the most essential one $[4,5]$ for the nucleon, whereas higher Fock states come next [6]. The LCWF in the context of quantum chromodynamics (QCD) is deeply rooted in the factorization theorem. It represents the soft part that is crucial in describing any high-energy processes. In general, the nonperturbative observables such as generalized parton distributions (GPDs), the parton distributions functions ( $\mathrm{PDFs}$ ), form factors $(\mathrm{FF})$, and fragmentation functions are formulated in terms of the hadronic matrix element of the non-local QCD operators, and all these quantities can be in principle constructed based on the LCWFs. For example, the 
GPDs [7-11], the PDFs [2, 12-15] and the transverse momentum-dependent parton distributions (TMDs) $[5,11,16]$ are accessed by the overlap representations of the LCWFs. Essential information on the LCWFs is contained in the distribution amplitudes (DAs), which encode how the quarks inside a hadron carry the fraction of the longitudinal momentum of the hadron. In this work, we want to investigate the DAs of the nucleon and $\Delta$ isobar.

The DAs have been intensively developed so that one can investigate the elastic or transition form factors at large momentum transfer for a meson [17-19] and a baryon [2, 3, 20,21]. They are formally defined as a vacuum-to-baryon matrix element of a nonlocal operator [3]. Since the contributions from the higher Fock components are power-suppressed, they are less important at larger momentum transfer. The form factors can be approximated to the convolution of the hard kernel of an exclusive process with the involved DAs from the leading Fock state. We want to mention that in the valence quark configuration the leading-twist DAs are relevant to the LCWFs with the orbital angular momentum $L_{z}=0$, whereas the higher-twist ones are connected to those with both $L_{z}=0$ and $L_{z} \neq 0$.

There has been a great number of theoretical works on the DAs. The dynamical properties of the nucleon DAs were investigated by employing the QCD sum rules [22-29] (see also reviews $[3,30]$ ). The effects of explicit SU(3) symmetry breaking $[27,31]$ on the hyperon DAs and those form factors were evaluated in refs. [27, 32-34]. The formalism for the DAs of the baryon decuplet was first explored in the QCD sum rules and the perturbative QCD predictions of the cross sections $\gamma \gamma \rightarrow B \bar{B}$ were obtained in ref. [35] (see also refs. [36-38]). The higher-twist DAs were systematically studied for the nucleon in ref. [39] and were associated with the electromagnetic form factors in refs. [29, 40-42]. Lattice QCD also provides invaluable information on the baryon octet DAs [43-48]. The proton and Roper DAs were studied in the framework of Dyson-Schwinger equations [49, 50]. Moreover, various phenomenological approaches [12, 51-57] have been developed, the results from lattice QCD and QCD sum rule being taken into account.

In this paper, we aim at providing information on the nucleon and $\Delta$ baryon DAs, and the corresponding normalization constants to the leading-twist accuracy by employing the chiral quark-soliton model ( $\chi \mathrm{QSM})$. The $\chi \mathrm{QSM}$, motivated by large $N_{c}$ QCD $[58,59]$, is a pion mean-field theory. The presence of the $N_{c}$ valence quarks creates the pion mean field, which comes from the vacuum polarization of the Dirac continuum. The $N_{c}$ valence quarks are then self-consistently influenced by this pion mean field. As a result, a baryon appears as a chiral soliton that consists of the $N_{c}$ valence quarks bound by the pion mean field. The $\chi$ QSM has been developed [60-62], based on the QCD instanton vacuum [63, 64]. It respects important properties of the low-energy QCD such as chiral symmetry and its spontaneous breaking. The $\chi$ QSM was successful in describing numerous low-energy properties of the baryon [62, 65]. In addition, the time-dependent mean fields describing the moving soliton with velocity $v \rightarrow 1$ are obtained from the stationary mean fields by the Lorentz boost and are applied to the nucleon PDFs [66-71] and GPDs [72, 73]. These PDFs and GPDs from the $\chi$ QSM comply with all general theorems and sum rules. In fact, the nucleon LCWF was first derived in ref. [74] in this framework and its large- $N_{c}$ features were studied in refs. [75, 76]. Based on the baryon LCWFs from the model, various observables [11, 77-83] were scrutinized by means of the overlap integrals. In this context, it is of great importance 
to examine the nucleon and $\Delta$ baryon DAs, which yield yet another essential information on the internal structures of the nucleon and $\Delta$ baryon.

We sketch the present work as follows: in section 2 we briefly review the definition of the nucleon DAs from the vacuum-to-nucleon matrix element of the trilocal QCD operator and discuss the symmetry properties of the nucleon DAs. In section 3 , the $\Delta$ baryon DAs is also derived in terms of the vacuum-to- $\Delta$ matrix element, and their symmetry properties are examined. The formalism for the LCWFs and DAs of the nucleon and $\Delta$ baryon is constructed within the framework of the $\chi \mathrm{QSM}$ in section 4 . The numerical results are presented and discussed in section 5. The final section 6 is devoted to summary and conclusions.

\section{Nucleon distribution amplitudes}

We start with a brief review on the general decomposition of the vacuum-to-nucleon matrix element of the trilocal quark field operators. It involves 24 different invariant functions, which was first investigated in ref. [39]. The matrix element of the three-quark operator on the light cone reads

$$
\left\langle 0\left|\epsilon^{i j k} u_{\alpha}^{i^{\prime}}\left(a_{1} n\right)\left[a_{1}, a_{0}\right]_{i^{\prime} i} u_{\beta}^{j^{\prime}}\left(a_{2} n\right)\left[a_{2}, a_{0}\right]_{j^{\prime} j} d_{\gamma}^{k^{\prime}}\left(a_{3} n\right)\left[a_{3}, a_{0}\right]_{k^{\prime} k}\right| N^{+}\left(p_{N}, \lambda\right)\right\rangle,
$$

where $\left|N^{+}\left(p_{N}, \lambda\right)\right\rangle$ stands for the proton state with its momentum $p_{N}\left(p_{N}^{2}=M_{N}^{2}\right)$ and helicity $\lambda$, respectively. The Greek letters $\alpha, \beta, \gamma$ denote the Dirac indices, whereas the Latin ones $i, j, k$ designate the color indices. $n$ represents an arbitrary light-like vector $\left(n^{2}=0\right)$ and $a_{i}$ is specified as the quark separation between quarks. In order to be a gauge-invariant matrix element, the gauge connection $\left[z_{i}, z_{0}\right]$ should be introduced:

$$
\left[a_{i}, a_{0}\right]=P \exp \left[i g\left(a_{i}-a_{0}\right) \int_{0}^{1} d t n_{\mu} A^{\mu}\left(n\left[t a_{i}+(1-t) a_{0}\right]\right)\right],
$$

where $P$ stands for the path-ordering. Since we choose the light-cone gauge $n \cdot A=0$, however, the gauge connection becomes the identity. Considering the explicit Lorentzcovariant parametrization with parity symmetry and the spin of the baryon taken into account, we are able to sort out the 24 different invariant functions, which are reduced to the three leading-twist ones:

$$
\begin{aligned}
& 4\left\langle 0\left|\epsilon^{i j k} u_{\alpha}^{i}\left(a_{1} n\right) u_{\beta}^{j}\left(a_{2} n\right) d_{\gamma}^{k}\left(a_{3} n\right)\right| N^{+}\left(p_{N}, \lambda\right)\right\rangle \\
& =f_{N}\left[\left(\not p_{N} C\right)_{\alpha \beta}\left(\gamma_{5} N\right)_{\gamma} V_{N}\left(a_{i} n \cdot p_{N}\right)+\left(\not p_{N} \gamma_{5} C\right)_{\alpha \beta} N_{\gamma} A_{N}\left(a_{i} n \cdot p_{N}\right)\right. \\
& \left.\quad+\left(i \sigma_{\mu \nu} p_{N}^{\nu} C\right)_{\alpha \beta}\left(\gamma^{\mu} \gamma_{5} N\right)_{\gamma} T_{N}\left(a_{i} n \cdot p_{N}\right)\right],
\end{aligned}
$$

where $C$ is the charge conjugation matrix and $\sigma_{\mu \nu}=i\left[\gamma_{\mu}, \gamma_{\nu}\right] / 2 . N$ is the nucleon spinor that satisfies the Dirac equation, i.e., $\not{ }_{N} N=M_{N} N$, and is normalized to be $\bar{N} N=2 M_{N}$. $f_{N}$ is known to be the nucleon decay constant or the DA normalization constant, which is identical to the value of the matrix element at the origin, given in eq. (2.3). The dimensionless functions $V_{N}, A_{N}$ and $T_{N}$ are the Lorentz scalars that depend on $p_{N} \cdot a_{i}$, and are normalized to be

$$
V_{N}(0)=T_{N}(0)=1, \quad A_{N}(0)=0 .
$$


In the leading-twist accuracy, the nucleon momentum $p_{N}^{\mu}$ can be approximated to the lightcone vector. The scalar functions $F=V_{N}\left(A_{N}, T_{N}\right)$ can be defined in the momentum space by the Fourier transforms:

$$
\begin{aligned}
F\left(x_{i}\right) \delta\left(1-\sum_{l=1}^{3} x_{l}\right) & =\left(n \cdot p_{N}\right)^{3} \int \prod_{j=1}^{3} \frac{d a_{j}}{(2 \pi)^{3}} F\left(a_{i} n \cdot p_{N}\right) \exp \left[i x_{k} a_{k}\left(n \cdot p_{N}\right)\right], \\
F\left(a_{i} n \cdot p_{N}\right) & =\int \prod_{j=1}^{3} d x_{j} \delta\left(1-\sum_{l=1}^{3} x_{l}\right) F\left(x_{i}\right) \exp \left[-i x_{k} a_{k}\left(n \cdot p_{N}\right)\right],
\end{aligned}
$$

where the variables $x_{i}$ denote the fractions of the baryon longitudinal momentum, carried by the partons on the light cone. They satisfy $\sum_{i=1}^{3} x_{i}=1$ and $0 \leq x_{i} \leq 1$ by momentum conservation. The DAs $V_{N}, A_{N}$, and $T_{N}\left(x_{i}\right)$ are defined at a certain scale $\mu$. For simplicity, we suppress it.

Not all the three scalar functions are independent. Exchanging the first two quarks and considering the properties of the transpose of the Dirac matrices in eq. (2.3), we find that $V_{N}, T_{N}$ are symmetric whereas $A_{N}$ becomes antisymmetric:

$$
\begin{aligned}
& V_{N}\left(x_{1}, x_{2}, x_{3}\right)=V_{N}\left(x_{2}, x_{1}, x_{3}\right), \quad A_{N}\left(x_{1}, x_{2}, x_{3}\right)=-A_{N}\left(x_{2}, x_{1}, x_{3}\right), \\
& T_{N}\left(x_{1}, x_{2}, x_{3}\right)=T_{N}\left(x_{2}, x_{1}, x_{3}\right) .
\end{aligned}
$$

In addition, we impose the requirement on the three coupled quarks to give an isospin $1 / 2$ state for the nucleon, which yields the following relation

$$
2 T_{N}\left(x_{1}, x_{2}, x_{3}\right)=[V-A]_{N}\left(x_{1}, x_{3}, x_{2}\right)+[V-A]_{N}\left(x_{2}, x_{3}, x_{1}\right) .
$$

Thus, we are able to express the nucleon DAs in terms of the single scalar function defined as $\varphi_{N}\left(x_{1}, x_{2}, x_{3}\right):=[V-A]_{N}\left(x_{1}, x_{2}, x_{3}\right)$. In flavor $\mathrm{SU}(3)$ symmetry, the given relation is valid for the baryon octet except for the isosinglet $\Lambda$ baryon. Therefore, all the hyperon DAs can be obtained by considering flavor SU(3) symmetry with the standard phase convention.

Suppressing the transverse-momentum dependence and considering only the $S$-wave $\left(L_{z}=0\right)$ contribution related to the leading-twist DAs, one finds that eq. (2.3) is equivalent to the given proton LCWF

$$
\begin{aligned}
\left|N^{+}\left(p_{N}, 1 / 2\right)\right\rangle=\frac{f_{N}}{8 \sqrt{6}} \int\left[\frac{d x}{\sqrt{x}}\right]_{3}[ & {[V-A]_{N}\left(x_{i}\right)\left|u^{\uparrow} u^{\downarrow} d^{\uparrow}\right\rangle+[V+A]_{N}\left(x_{i}\right)\left|u^{\downarrow} u^{\uparrow} d^{\uparrow}\right\rangle } \\
& \left.-T_{N}\left(x_{i}\right)\left|u^{\uparrow} u^{\uparrow} d^{\downarrow}\right\rangle\right],
\end{aligned}
$$

where the integration measure and the three quark states are respectively defined as

$$
\begin{aligned}
\int\left[\frac{d x}{\sqrt{x}}\right]_{n} & :=\int\left[\prod_{j=1}^{n} \frac{d x_{j}}{\sqrt{x_{j}}}\right] \delta\left(1-\sum_{l=1}^{n} x_{l}\right), \\
\left|f^{\sigma_{1}} g^{\sigma_{2}} h^{\sigma_{3}}\right\rangle & =\frac{\epsilon^{\alpha_{1} \alpha_{2} \alpha_{3}}}{\sqrt{6}} a_{\alpha_{1} f \sigma_{1}}^{\dagger}\left(\boldsymbol{p}_{1}\right) a_{\alpha_{2} g \sigma_{2}}^{\dagger}\left(\boldsymbol{p}_{2}\right) a_{\alpha_{3} h \sigma_{3}}^{\dagger}\left(\boldsymbol{p}_{3}\right)|0\rangle .
\end{aligned}
$$


The three-quark states carry the color $(\alpha=1,2,3)$, flavor $(f=1,2,3=u, d, s)$ and spin projection $(\sigma=1,2=\uparrow, \downarrow)$ indices. Here the nucleon state, with the quantum numbers suppressed, is normalized as

$$
\left\langle N(p) \mid N\left(p^{\prime}\right)\right\rangle=2 p^{+}(2 \pi)^{3} \delta\left(p^{+}-p^{\prime+}\right) \delta^{(2)}\left(\boldsymbol{p}_{\perp}-\boldsymbol{p}_{\perp}^{\prime}\right)
$$

with the light-cone momenta $p^{ \pm}=\left(p_{0} \pm p_{z}\right) / \sqrt{2}$.

\section{$3 \quad \Delta$ baryon distribution amplitudes}

We now examine the properties of the $\Delta$-baryon DAs. The general decomposition of the vacuum-to- $\Delta$ matrix element of the trilocal quark field operators has been derived in ref. [35]. The general decomposition of the corresponding matrix element in the leading twist is obtained to be

$$
\begin{aligned}
& 4\left\langle 0\left|\epsilon^{i j k} u_{\alpha}^{i}\left(a_{1} n\right) u_{\beta}^{j}\left(a_{2} n\right) u_{\gamma}^{k}\left(a_{3} n\right)\right| \Delta^{++}\left(p_{\Delta}, \lambda\right)\right\rangle \\
& =\lambda_{\Delta}^{1 / 2}\left[\left(\gamma_{\mu} C\right)_{\alpha \beta} \Delta_{\gamma}^{\mu} V_{\Delta}\left(a_{i} n \cdot p_{\Delta}\right)+\left(\gamma_{\mu} \gamma_{5} C\right)_{\alpha \beta}\left(\gamma_{5} \Delta^{\mu}\right)_{\gamma} A_{\Delta}\left(a_{i} n \cdot p_{\Delta}\right)\right. \\
& \left.-\frac{1}{2}\left(i \sigma_{\mu \nu} C\right)_{\alpha \beta}\left(\gamma^{\mu} \Delta^{\nu}\right)_{\gamma} T_{\Delta}\left(a_{i} n \cdot p_{\Delta}\right)\right] \\
& -f_{\Delta}^{3 / 2}\left[\left(i \sigma_{\mu \nu} C\right)_{\alpha \beta}\left(p^{\mu} \Delta^{\nu}-\frac{1}{2} M_{\Delta} \gamma^{\mu} \Delta^{\nu}\right)_{\gamma} \varphi_{\Delta}^{3 / 2}\left(a_{i} n \cdot p_{\Delta}\right)\right],
\end{aligned}
$$

where $\Delta^{\mu}\left(p_{\Delta}, \lambda\right)$ denotes the Rarita-Schwinger spinor that satisfies

$$
\left(\not p_{\Delta}-M_{\Delta}\right) \Delta^{\mu}\left(p_{\Delta}, \lambda\right)=0, \quad \bar{\Delta}^{\mu} \Delta_{\mu}=-2 M_{\Delta}, \quad \gamma_{\mu} \Delta^{\mu}(p, \lambda)=p_{\Delta}^{\mu} \Delta_{\mu}\left(p_{\Delta}, \lambda\right)=0 .
$$

$f_{\Delta}^{1 / 2}=\sqrt{2 / 3} \lambda_{\Delta}^{1 / 2} / M_{\Delta}$ and $f_{\Delta}^{3 / 2}$ represent the $\Delta$ baryon decay constants or the DA normalization constants that is equivalent to the value of the vacuum-to- $\Delta$ matrix element at the origin. The Lorentz scalar functions $V_{\Delta}, A_{\Delta}, T_{\Delta}$ and $\varphi_{\Delta}^{3 / 2}$ are therefore normalized to be

$$
V_{\Delta}(0)=T_{\Delta}(0)=\varphi_{\Delta}^{3 / 2}(0)=1, \quad A_{\Delta}(0)=0 .
$$

The $\Delta$ baryon DAs satisfy the following symmetries:

$$
\begin{aligned}
& V_{\Delta}\left(x_{1}, x_{2}, x_{3}\right)=V_{\Delta}\left(x_{2}, x_{1}, x_{3}\right), \quad A_{\Delta}\left(x_{1}, x_{2}, x_{3}\right)=-A_{\Delta}\left(x_{2}, x_{1}, x_{3}\right), \\
& T_{\Delta}\left(x_{1}, x_{2}, x_{3}\right)=T_{\Delta}\left(x_{2}, x_{1}, x_{3}\right) .
\end{aligned}
$$

and

$$
T_{\Delta}\left(x_{1}, x_{2}, x_{3}\right)=[V-A]_{\Delta}\left(x_{2}, x_{3}, x_{1}\right) .
$$

Note that $\varphi_{\Delta}^{3 / 2}\left(x_{1}, x_{2}, x_{3}\right)$ is found to be totally symmetric under the exchange of its variables. Using these symmetries, we can express the $\Delta$ DAs in terms of the single DA $\varphi_{\Delta}^{1 / 2}\left(x_{1}, x_{2}, x_{3}\right) \equiv[V-A]_{\Delta}\left(x_{1}, x_{2}, x_{3}\right)$ for spin projection $1 / 2$ and $\varphi_{\Delta}^{3 / 2}\left(x_{1}, x_{2}, x_{3}\right)$ for spin projection $3 / 2$. Suppressing again the representation of the transversal momentum, we arrive at the $\Delta^{++}$LCWF for $\lambda=3 / 2$

$$
\left|\Delta\left(p_{\Delta}, 3 / 2\right)\right\rangle=-\frac{f_{\Delta}^{3 / 2}}{24 \sqrt{3}} \int\left[\frac{d x}{\sqrt{x}}\right]_{3}\left[\varphi_{\Delta}^{3 / 2}\left(x_{i}\right)\left|u^{\uparrow} u^{\uparrow} u^{\uparrow}\right\rangle\right],
$$


and for $\lambda=1 / 2$

$$
\begin{aligned}
\left|\Delta\left(p_{\Delta}, 1 / 2\right)\right\rangle=-\frac{f_{\Delta}^{1 / 2}}{24 \sqrt{6}} \int\left[\frac{d x}{\sqrt{x}}\right]_{3}[ & {[V-A]_{\Delta}\left(x_{i}\right)\left|u^{\uparrow} u^{\downarrow} u^{\uparrow}\right\rangle+[V+A]_{\Delta}\left(x_{i}\right)\left|u^{\downarrow} u^{\uparrow} u^{\uparrow}\right\rangle } \\
& \left.+T_{\Delta}\left(x_{i}\right)\left|u^{\uparrow} u^{\uparrow} u^{\downarrow}\right\rangle\right] .
\end{aligned}
$$

\section{Light-cone wave function in the chiral quark-soliton model}

The effective chiral Lagrangian $[60,62,64]$ is expressed as

$$
\mathcal{L}_{\text {eff }}=\bar{\psi}(x)\left(i \not \partial-M U^{\gamma_{5}}\right) \psi(x),
$$

where $\psi$ and $U^{\gamma_{5}}$ stand for the quark and chiral fields respectively. The $M$ represents the dynamical quark mass. In principle, the dynamical quark mass is originally the momentumdependent one, i.e. $M(p)$ that can be derived from the zero-mode fermionic solution in the QCD instanton vacuum $[64,84,85]$. This plays a role of the natural regulator for a quark loop. In this work, we turn off the momentum dependence of the dynamical quark mass for simplicity and introduce an explicit regularization scheme. We use the Pauli-Villars method. The SU(2) chiral field is defined as:

$$
U^{\gamma_{5}}=\frac{1+\gamma_{5}}{2} U+\frac{1-\gamma_{5}}{2} U^{\dagger}, \quad U=\exp \left(i \pi^{a}(x) \tau^{a}\right),
$$

where $\pi^{a}(x)$ are the pseudo-Nambu-Goldstone (pNG) boson fields. The pNG field has a hedgehog symmetry, which is the minimum generalization of spherical symmetry [86]:

$$
\pi(\boldsymbol{x})=n^{a} P(r), \quad \text { with } \quad n^{a}=x^{a} /|\boldsymbol{x}| .
$$

The nucleon mass can be derived from the nucleon correlation function, which is defined as

$$
\Pi(T)=\left\langle 0\left|J_{N}(T / 2, \mathbf{0}) J_{N}^{\dagger}(-T / 2, \mathbf{0})\right| 0\right\rangle,
$$

where the $J_{N}$ is the Ioffe-type current carrying the quantum numbers of the nucleon

$$
J_{N}(t, \boldsymbol{x})=\frac{1}{N_{c} !} \epsilon^{\beta_{1} \ldots \beta_{N_{c}}} \Gamma_{J J_{3}, I_{3}}^{\{f\}} \Psi_{\beta_{1} f_{1}}(t, \boldsymbol{x}) \ldots \Psi_{\beta_{N_{c}} f_{N_{c}}}(t, \boldsymbol{x}) .
$$

Here, the $\beta_{1}, \cdots, \beta_{N_{c}}$ denote the color indices and $\Gamma^{\{f\}}$ is a matrix with spin-flavor indices $f$. The $J$ and $I$ represent respectively the nucleon spin and isospin quantum numbers, and $J_{3}$ and $I_{3}$ their third components, respectively. At the large Euclidean time separation, i.e., $T \rightarrow \infty$, the correlation function yields the nucleon mass $M_{N}$ :

$$
\lim _{T \rightarrow \infty} \Pi_{N}(T) \sim e^{-M_{N} T},
$$

where it is evaluated by the functional integral over the quark fields. Since the functional integral over the chiral field cannot be carried out exactly, an approximation should be made. In the limit of large $N_{c}$, we are able to use the saddle point approximation for the integral 
over $U$. In this approximation, one obtains the classical soliton mass by minimizing the energy around the saddle point of the pion mean field, which describes the classical nucleon

$$
\left.\frac{\delta M_{N}[U]}{\delta U}\right|_{U=U_{c}}=0
$$

and the quantum fluctuation of the pion field is suppressed by $1 / N_{c}$. Thus, we restrict ourselves to the leading order of $N_{c}$. The self-consistent pionic configuration $U_{c}(\boldsymbol{x})$ and the classical soliton mass $M_{N}=1.207 \mathrm{GeV}$ were obtained in ref. [87], and the corresponding results are well approximated by the given arctangent-type profile function

$$
P(r)=2 \arctan \left(\frac{r_{0}^{2}}{r^{2}}\right), \quad r_{0}=\frac{0.8}{M}, \quad M=0.345 \mathrm{GeV},
$$

which is depicted in the left panel of figure 1 . Note that the masses of the nucleon and $\Delta$ baryon are degenerate at the classical level.

\subsection{Baryon wave functions}

To construct the baryon wave function, we need to calculate the finite-time evolution operator with the definite boundary condition within the functional integral [88]. In the large $N_{c}$ limit, the wave function of the classical nucleon (soliton), completely factorized in the color space, can be obtained by applying the finite-time evolution operator to any color singlet state of the $N_{c}$ valence quarks and by taking the finite time $T$ to be infinity $[74,77]$ :

$$
|\Psi\rangle=\prod_{\text {color }} \int \frac{d^{3} \boldsymbol{p}}{(2 \pi)^{3}} F(\boldsymbol{p}) a^{\dagger}(\boldsymbol{p})|\Omega\rangle,
$$

where the vacuum wave function or Dirac sea $|\Omega\rangle$ is given by the quark and anti-quark creation operators

$$
|\Omega\rangle=\exp \left[\sum_{\text {color }} \int \frac{d^{3} \boldsymbol{p}}{(2 \pi)^{3}} \frac{d^{3} \boldsymbol{p}^{\prime}}{(2 \pi)^{3}} a^{\dagger}(\boldsymbol{p}) W\left(\boldsymbol{p}, \boldsymbol{p}^{\prime}\right) b^{\dagger}\left(\boldsymbol{p}^{\prime}\right)\right]|0\rangle,
$$

with the quark-antiquark pair wave function $W\left(\boldsymbol{p}, \boldsymbol{p}^{\prime}\right)$

$$
W\left(\boldsymbol{p}, \boldsymbol{p}^{\prime}\right)=-i \sqrt{\frac{\varepsilon \varepsilon^{\prime}}{M^{2}}} \bar{u}(\boldsymbol{p}) G\left(\boldsymbol{p}, T, \boldsymbol{p}^{\prime}, T\right) v\left(\boldsymbol{p}^{\prime}\right) .
$$

The $\bar{u}$ and $v$ denote the Dirac spinors normalized to be $\bar{u} u=-\bar{v} v=1$ and $\epsilon=\sqrt{\boldsymbol{p}^{2}+M^{2}}$. The creation and annihilation operators $a^{\dagger}\left(b^{\dagger}\right)$ and $a(b)$ for a quark (antiquark) satisfy the usual anticommutation relation

$$
\left\{a\left(\boldsymbol{p}^{\prime}\right), a^{\dagger}(\boldsymbol{p})\right\}=(2 \pi)^{3} \delta^{(3)}\left(\boldsymbol{p}^{\prime}-\boldsymbol{p}\right), \quad\left\{b\left(\boldsymbol{p}^{\prime}\right), b^{\dagger}(\boldsymbol{p})\right\}=(2 \pi)^{3} \delta^{(3)}\left(\boldsymbol{p}^{\prime}-\boldsymbol{p}\right) .
$$

The vacuum state can be annihilated as follows: $a|0\rangle=0, b|0\rangle=0,\langle 0| a^{\dagger}=0$, and $\langle 0| b^{\dagger}=0$. The quark annihilation-creation operators carry the color $\alpha$, flavor $f$ and spin projection $\sigma$ indices, including the three-momentum $\boldsymbol{p}$. However, we will suppress them except for 
the momentum from now on. The Green function in the presence of the pion mean field in eq. (4.11) and its Fourier transform are defined as

$$
\begin{aligned}
G\left(\boldsymbol{p}, T, \boldsymbol{p}^{\prime}, T\right) & :=\int d^{3} \boldsymbol{x} d^{3} \boldsymbol{y} e^{-i \boldsymbol{p} \cdot \boldsymbol{x}} e^{-i \boldsymbol{p}^{\prime} \cdot \boldsymbol{y}} G(\boldsymbol{x}, T, \boldsymbol{y}, T) \\
\text { with } \quad\left(i \not \partial-M U^{\gamma_{5}}\right) G(x, y) & =\delta^{(4)}(x-y) .
\end{aligned}
$$

$F(\boldsymbol{p})$ denotes the valence quark wave function, defined as

$$
\begin{aligned}
F(\boldsymbol{p})=\int \frac{d^{3} \boldsymbol{p}^{\prime}}{(2 \pi)^{3}} \sqrt{\frac{M}{\varepsilon^{\prime}}}[ & \bar{u}(\boldsymbol{p}) \gamma^{0} \psi_{\mathrm{lev}}(\boldsymbol{p})(2 \pi)^{3} \delta^{(3)}\left(\boldsymbol{p}-\boldsymbol{p}^{\prime}\right) \\
& \left.-W\left(\boldsymbol{p}, \boldsymbol{p}^{\prime}\right) \bar{v}\left(\boldsymbol{p}^{\prime}\right) \gamma^{0} \psi_{\mathrm{lev}}\left(-\boldsymbol{p}^{\prime}\right)\right]
\end{aligned}
$$

which consists of two parts: the first term corresponds to the discrete-level wave function whereas the second term arises from the distortion of this wave function, which is exerted by the vacuum wave function. In this work, the second term is ignored bacause of its complexity. Note that its effects on observables were estimated in refs. [11, 74] in the accuracy of the $3 Q$ Fock component and yielded about $10 \%$ contributions. The $\psi_{\text {lev }}$ stands for the discrete-level wave function, satisfying the Dirac equation in the presence of the external chiral field with the eigenenergies $E_{\text {lev }}$ in the $K^{p}=0^{+}$sector with $\boldsymbol{K}=\boldsymbol{I}+\boldsymbol{J}$. $\boldsymbol{J}$ and $\boldsymbol{I}$ denote the spin and isospin rotation generators, respectively. It is derived as

$$
\psi_{\mathrm{lev}}(r)=\left(\begin{array}{c}
\epsilon^{j i} h(r) \\
-i \epsilon^{j k}(\sigma \cdot \boldsymbol{n})_{k}^{i} j(r)
\end{array}\right), \quad\left\{\begin{array}{c}
h^{\prime}+h M \sin P-j\left(M \cos P+E_{\mathrm{lev}}\right)=0 \\
j^{\prime}+2 j / r-j M \sin P-h\left(M \cos P-E_{\mathrm{lev}}\right)=0
\end{array},\right.
$$

where $i=1,2=\uparrow, \downarrow$ denotes the spin projection index and $j=1,2=u, d$ stands for the isospin index. Inserting the self-consistent field (4.8) into eq. (4.15), one obtains the eigenenergy $E_{\text {lev }}=0.2 \mathrm{GeV}$ and the discrete-level wave function $\psi_{\text {lev }}(r)$ of the valence quark, which is tightly bound by the self-consistent fields. In the nonrelativistic limit, the upper component $(L=0)$ of the Dirac bispinor, $h(r)$, is dominant whereas its lower component $(L=1) j(r) \sim 0$ is suppressed. $\psi_{\text {lev }}(\boldsymbol{p})$ in eq. (4.14) is the Fourier transform of eq. (4.15). The discrete-level wave functions are plotted in the right panel of figure 1.

\subsection{Quantization}

The baryon wave function given in eq. (4.9) is still a classical one. While the quantum fluctuations of the pion fields are completely ignored on account of the $N_{c}$ counting, the fluctuations in the direction of the translational and rotational zero modes should be considered in an exact manner. By this zero-mode quantization, we are able to construct the baryon state that acquires the momentum conservation and the correct quantum numbers $[60,62,64]$. As a result, the quantized baryon wave function is given by

$$
\begin{aligned}
\left|\Psi^{k}(B)\right\rangle= & \int d R B_{k}^{*}(R) \frac{\epsilon^{\alpha_{1} \alpha_{2} \alpha_{3}}}{\sqrt{N_{c} !}}\left[\prod_{n=1}^{N_{c}} \int \frac{d^{3} \boldsymbol{p}_{n}}{(2 \pi)^{3}} R_{j_{n}}^{f_{n}} F^{j_{n} \sigma_{n}}\left(\boldsymbol{p}_{n}\right) a_{\alpha_{n} f_{n} \sigma_{n}}^{\dagger}\left(\boldsymbol{p}_{n}\right)\right] \\
& \times \exp \left(\int \frac{d^{3} \boldsymbol{p}}{(2 \pi)^{3}} \frac{d^{3} \boldsymbol{p}^{\prime}}{(2 \pi)^{3}} a_{\alpha f \sigma}^{\dagger}(\boldsymbol{p}) R_{j}^{f} W_{j^{\prime} \sigma^{\prime}}^{j \sigma}\left(\boldsymbol{p}, \boldsymbol{p}^{\prime}\right) R_{j^{\prime}}^{\dagger f^{\prime}} b^{\dagger \alpha f^{\prime} \sigma^{\prime}}\left(\boldsymbol{p}^{\prime}\right)\right)|0\rangle,
\end{aligned}
$$



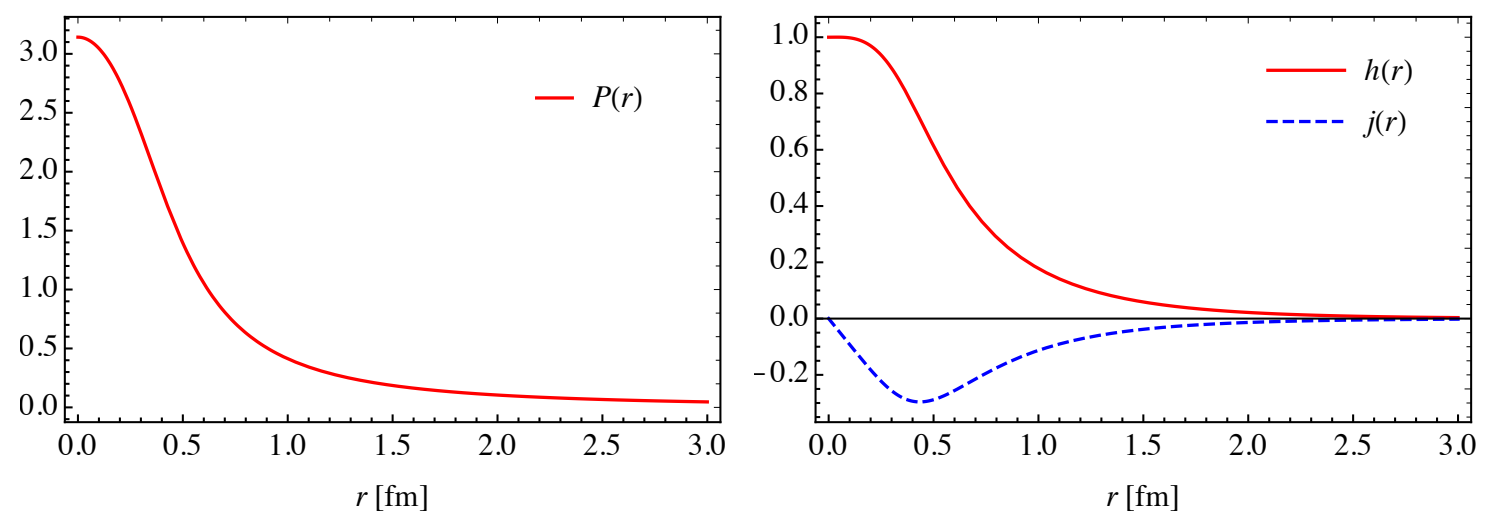

Figure 1. The left panel depicts the self-consistent profile function, approximated to the arctangent one, as a function of $r$ whereas the right panel draws the discrete-level wave functions $h$ and $j$ as a function of $r$ with the boundary condition $h(0)=1$ imposed.

where $k$ denotes the spin projection of the baryon state. The color $(\alpha=1,2,3)$, flavor $(f=1,2,3)$, isospin $(j=1,2)$ and spin projection $(\sigma=1,2)$ indices suppressed in eq. (4.9) are explicitly restored.

The higher Fock components are generated by expanding the vacuum wave function $|\Omega\rangle$ to the order of what we are interested in, i.e., $|\Omega\rangle=e^{\bar{q} q} \sim 1+\bar{q} q+\ldots$. Integration over the rotation matrices $R_{j}^{f}$ projects the flavor state of all valence quarks and quark-antiquark out of the vacuum wave function onto the spin-flavor state $B^{*}(R)$, which describes the baryon octet and decuplet. While this state can be expressed by the $\mathrm{SU}(3)$ Wigner $D$ functions [89], which is often evaluated in terms of the SU(3) Clebsch-Gordan coefficients, it is more convenient to integrate over the Haar measure $d R$ to obtain them. This method has a great virtue that the symmetries of the quark wave functions are revealed naturally. The explicit expressions of the baryon rotational wave functions are given in appendix A. Note that the third row of the matrix $R_{j}^{f}, f=3$ makes it possible to pop up the strange quarks both at the valence level and in the Dirac sea. The physical baryon state is then decomposed into the Fock components:

$$
\left|\Psi^{k}(B)\right\rangle=\left|\Psi^{(3) k}(B)\right\rangle+\left|\Psi^{(5) k}(B)\right\rangle+\ldots,
$$

where

$$
\left\langle\Psi^{k}(B) \mid \Psi^{k}(B)\right\rangle=\mathcal{N}^{(3)}+\mathcal{N}^{(5)} \ldots, \quad\left\langle\Psi^{(n) k}(B) \mid \Psi^{(n) k}(B)\right\rangle=\mathcal{N}^{(n)} .
$$

The baryon state is normalized by the factor $\sqrt{\mathcal{N}^{(3)}+\mathcal{N}^{(5)} \ldots}$, which ensures that it should be normalized to be unity (4.18). In principle, while all possible higher Fock components should be taken into account for normalization, it is not possible to consider them in practice. Thus, we need to truncate the higher Fock states at a certain level. In this work, we include the $3 Q$, and $5 Q$ components.

\subsection{Baryon light-cone wave functions}

We are now in a position to derive the LCWFs for the baryon. The LCWF of the baryon is defined on the light cone. While the stationary saddle-point solution $U_{c}(\boldsymbol{x})$ corresponds to 

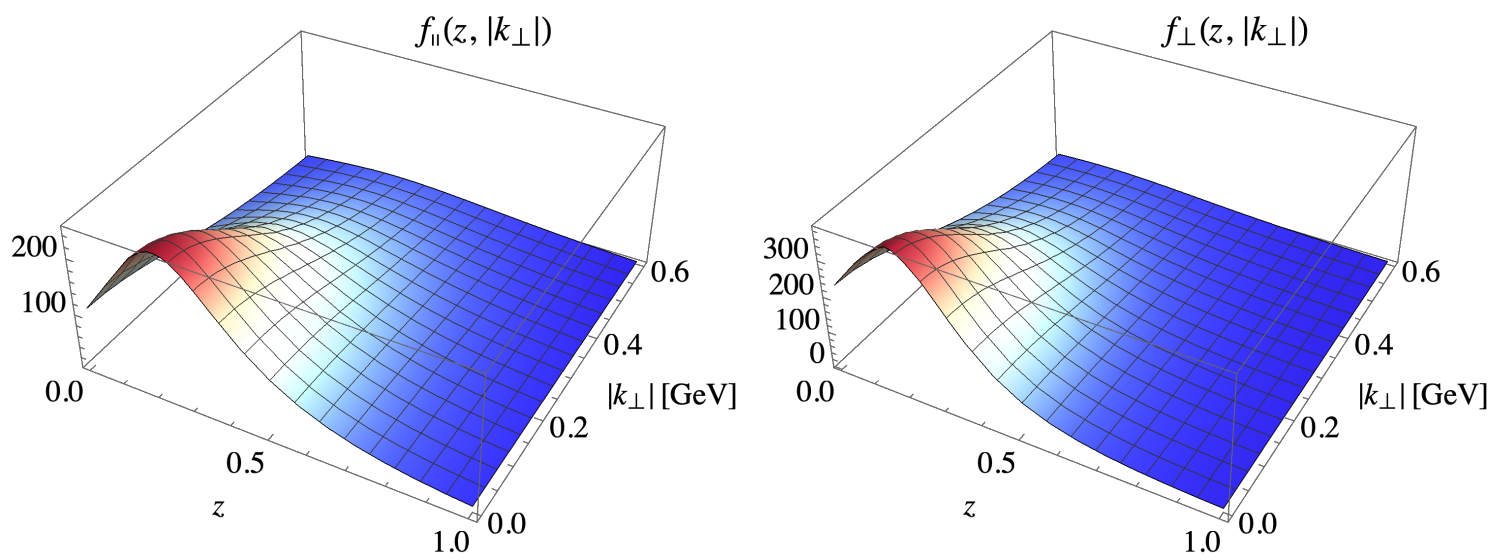

Figure 2. The two independent components $f_{\|}\left(z,\left|\boldsymbol{k}_{\perp}\right|\right)$ and $f_{\perp}\left(z,\left|\boldsymbol{k}_{\perp}\right|\right)$ are drawn on the light cone.

the classical baryon at rest, it is necessary to construct the pion mean field that describes the moving baryon, i.e., $U(t, \boldsymbol{x})$, with its velocity $v \rightarrow 1$. Since the effective chiral Lagrangian is Lorentz invariant, it is straightforward to boost the baryon to the light cone. This means that we can easily obtain the discrete-level wave function and the self-consistent mean field on the light cone by the Lorentz boost. Taking the velocity $v \rightarrow 1$, we derive the valence-quark wave function

$$
F^{j \sigma}\left(z, \boldsymbol{p}_{\perp}\right)=\left.\left(\begin{array}{cc}
k_{L} f_{\perp}\left(z,\left|\boldsymbol{k}_{\perp}\right|\right) & f_{\|}\left(z,\left|\boldsymbol{k}_{\perp}\right|\right) \\
-f_{\|}\left(z,\left|\boldsymbol{k}_{\perp}\right|\right) & k_{R} f_{\perp}\left(z,\left|\boldsymbol{k}_{\perp}\right|\right)
\end{array}\right)^{j \sigma}\right|_{k_{z}=z M_{N}-E_{\mathrm{lev}}, \boldsymbol{k}_{\perp}=\boldsymbol{p}_{\perp}},
$$

with $k_{R, L}=k_{x} \pm i k_{y}$. The two indenpendent functions $f_{\|}\left(z,\left|\boldsymbol{k}_{\perp}\right|\right)$ and $f_{\perp}\left(z,\left|\boldsymbol{k}_{\perp}\right|\right)$ are written as

$$
f_{\|}\left(z,\left|\boldsymbol{k}_{\perp}\right|\right)=\sqrt{\frac{M_{N}}{2 \pi}}\left(h(k)+\frac{k_{z} j(k)}{|\boldsymbol{k}|}\right), \quad f_{\perp}\left(z,\left|\boldsymbol{k}_{\perp}\right|\right)=\sqrt{\frac{M_{N}}{2 \pi}} \frac{j(k)}{|\boldsymbol{k}|} .
$$

In figure 2, we illustrate $f_{\|}\left(z,\left|\boldsymbol{k}_{\perp}\right|\right)$ and $f_{\perp}\left(z,\left|\boldsymbol{k}_{\perp}\right|\right)$ on the light cone. Note that in the nonrelativistic limit the function $f_{\|}^{\mathrm{NR}}$ is solely responsible for the valence-quark wave function $F$ :

$$
f_{\|}^{\mathrm{NR}}\left(z,\left|\boldsymbol{k}_{\perp}\right|\right)=\sqrt{\frac{M_{N}}{2 \pi}} h(k), \quad f_{\perp}^{\mathrm{NR}}\left(z,\left|\boldsymbol{k}_{\perp}\right|\right)=0 .
$$

The creation and annihilation operators for the quarks satisfy the following anticommutation relation

$$
\left\{a_{\sigma}^{\dagger}\left(z, \boldsymbol{p}_{\perp}\right), a_{\sigma^{\prime}}\left(z^{\prime}, \boldsymbol{p}_{\perp}^{\prime}\right)\right\}=\delta_{\sigma \sigma^{\prime}} \delta\left(z-z^{\prime}\right)(2 \pi)^{2} \delta^{(2)}\left(\boldsymbol{p}_{\perp}-\boldsymbol{p}_{\perp}^{\prime}\right),
$$

so that the wave function is properly normalized. It is convenient to rescale the creation operators to be $a_{\sigma}\left(z, \boldsymbol{p}_{\perp}\right)=\sqrt{P_{N} / 2 \pi} a_{\sigma}(\boldsymbol{p})$.

The pair wave function $W\left(\boldsymbol{p}, \boldsymbol{p}^{\prime}\right)$ is expressed in terms of the finite-time Green function at an equal time in the presence of the self-consistent pion field. The Green function is the 


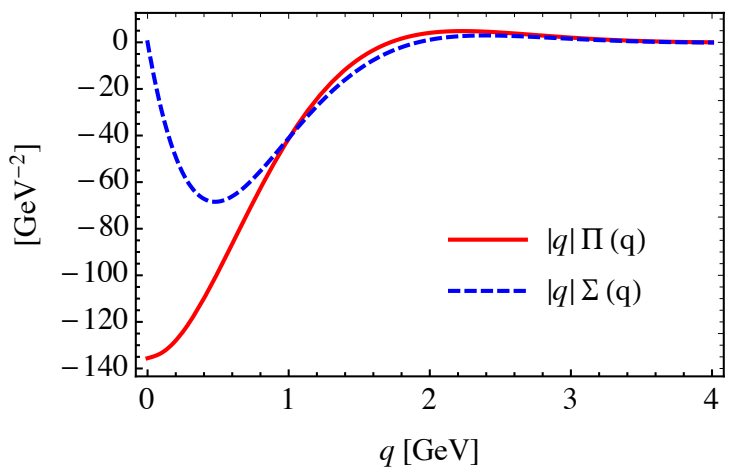

Figure 3. Scalar $(|\boldsymbol{q}| \Sigma(q))$ and pseudoscalar $(|\boldsymbol{q}| \Pi(q))$ mean fields are illustrated by the dashed and solid curves, respectively.

solution of eq. (4.13). The chiral field is decomposed into the scalar and pseudoscalar fields:

$$
U^{\gamma_{5}}=1+\Sigma+i \Pi \gamma_{5}
$$

where $U^{\gamma_{5}}$ is constrained on the chiral circle $(\Sigma+1)^{2}+\Pi^{2}=1$. These scalar and pseudoscalar fields in the momentum space are obtained by the Fourier transforms:

$$
\Pi(\boldsymbol{q})_{j^{\prime}}^{j}=\int d^{3} \boldsymbol{x} e^{-i \boldsymbol{q} \cdot \boldsymbol{x}}(\boldsymbol{n} \cdot \boldsymbol{\tau})_{j^{\prime}}^{j} \sin P(r), \quad \Sigma(\boldsymbol{q})_{j^{\prime}}^{j}=\int d^{3} \boldsymbol{x} e^{-i \boldsymbol{q} \cdot \boldsymbol{x}} \delta_{j^{\prime}}^{j}(\cos P(r)-1)
$$

where the pseudoscalar field $\Pi(\boldsymbol{q})$ is a purely imaginary and odd function, whereas the scalar field $\Sigma(\boldsymbol{q})$ is a real and even function. The explicit forms of these two fields are written as

$$
\begin{array}{ll}
\Pi(\boldsymbol{q})_{j^{\prime}}^{j}=i \frac{(\boldsymbol{q} \cdot \boldsymbol{\tau})_{j^{\prime}}^{j}}{|\boldsymbol{q}|} \Pi(q), & \Pi(q)=-\int d^{3} \boldsymbol{x} j_{1}(q r) \sin P(r), \\
\Sigma(\boldsymbol{q})_{j^{\prime}}^{j}=\delta_{j^{\prime}}^{j} \Sigma(q), & \Sigma(q)=\int d^{3} \boldsymbol{x} j_{0}(q r)(\cos P(r)-1) .
\end{array}
$$

$\Sigma(\boldsymbol{q})$ and $\Pi(\boldsymbol{q})$ are plotted in figure 3.

It is rather complicated to derive the quark-antiquark pair wave function $W\left(\boldsymbol{p}, \boldsymbol{p}^{\prime}\right)$ exactly. Hence, we confine ourselves to the interpolation approximation for it. Note that in the three limiting cases of the pion field, i.e., the small pion field $P(r)$, the slowly varying pion field, and fastly varying pion field, $W\left(\boldsymbol{p}, \boldsymbol{p}^{\prime}\right)$ becomes exact. Typically, the error between the interpolation approximation and the exact calculation lies at most within $15 \%$. By taking $v \rightarrow 1$, the quark-antiquark pair wave function (4.10) on the light cone is then obtained to be

$$
W_{j^{\prime} \sigma^{\prime}}^{j \sigma}\left(\boldsymbol{q}, y, \mathcal{Q}_{\perp}\right)=\frac{M_{N} M}{2 \pi} \frac{\sum_{j^{\prime}}^{j}(\boldsymbol{q})\left[M(2 y-1) \tau_{3}+\mathcal{Q}_{\perp} \cdot \boldsymbol{\tau}_{\perp}\right]_{\sigma^{\prime}}^{\sigma}+i \Pi_{j^{\prime}}^{j}(\boldsymbol{q})\left[-M \mathbf{1}+i \mathcal{Q}_{\perp} \times \boldsymbol{\tau}_{\perp}\right]_{\sigma^{\prime}}^{\sigma}}{\mathcal{Q}_{\perp}^{2}+M^{2}+y(1-y) \boldsymbol{q}^{2}}
$$

with

$$
y=\frac{z^{\prime}}{z+z^{\prime}}, \quad \mathcal{Q}_{\perp}=\frac{z \boldsymbol{p}_{\perp}^{\prime}-z^{\prime} \boldsymbol{p}_{\perp}}{z+z^{\prime}}, \quad \boldsymbol{q}=\left(\left(\boldsymbol{p}+\boldsymbol{p}^{\prime}\right)_{\perp}, M_{N}\left(z+z^{\prime}\right)\right)
$$




\subsection{Fock components of the baryon light-cone wave functions}

Various models for the baryon LCWFs mainly focus on the $3 Q$ Fock component, which is a rather crude approximation in reality. However, it was found in refs. [78, 81] that the higher Fock components are nonnegligible and come into significant play in studying the various features of the nucleon. The $5 Q$ Fock component provides at least $20 \%$ contribution to observables. Thus, we will derive both the $3 Q$ and $5 Q$ wave functions for the baryon octet and decuplet.

\subsection{1 $3 Q$ Fock component}

Expanding eq. (4.16) and taking its leading term, we derive the baryon LCWF of the $3 Q$ component

$$
\left|\Psi^{(3) k}(B)\right\rangle=T(B)_{j_{1} j_{2} j_{3}, k}^{f_{1} f_{2}} \frac{\epsilon^{\alpha_{1} \alpha_{2} \alpha_{3}}}{\sqrt{N_{c} !}} \prod_{n=1}^{N_{c}} \int \frac{d^{3} \boldsymbol{p}_{n}}{(2 \pi)^{3}} F^{j_{n} \sigma_{n}}\left(\boldsymbol{p}_{n}\right) a_{\alpha_{n} f_{n} \sigma_{n}}^{\dagger}\left(\boldsymbol{p}_{n}\right)|0\rangle .
$$

Each of three valence quarks is rotated by the matrix $R_{j}^{f}$ and projected onto the spinflavor baryon state $B^{*}(R)$ by integrating over $R$. The short-handed notation for this group integral is defined as

$$
T(B)_{j_{1} j_{2} j_{3}, k}^{f_{1} f_{2} f_{3}}:=\int d R B_{k}^{*}(R) R_{j_{1}}^{f_{1}} R_{j_{2}}^{f_{2}} R_{j_{3}}^{f_{3}} .
$$

We refer to refs. [78, 79, 81, 90] for details.

The normalization $\mathcal{N}^{(3)}$ of the baryon LCWF from eq. (4.18) is obtained by contracting the creation and annihilation operators, which is written as

$$
\begin{aligned}
\mathcal{N}^{(3)}(B)= & 6 T(B)_{j_{1} j_{2} j_{3}, k}^{f_{1} f_{2} f_{3}} T(B)_{f_{1} f_{2} f_{3}}^{j_{1}^{\prime} j^{\prime} j_{3}^{\prime}, k} \\
& \times \int[d \boldsymbol{p}]_{n} F^{j_{1} \sigma_{1}}\left(\boldsymbol{p}_{1}\right) F^{j_{2} \sigma_{2}}\left(\boldsymbol{p}_{2}\right) F^{j_{3} \sigma_{3}}\left(\boldsymbol{p}_{3}\right) F_{j_{1}^{\prime} \sigma_{1}}^{\dagger}\left(\boldsymbol{p}_{1}\right) F_{j_{2}^{\prime} \sigma_{2}}^{\dagger}\left(\boldsymbol{p}_{2}\right) F_{j_{3}^{\prime} \sigma_{3}}^{\dagger}\left(\boldsymbol{p}_{3}\right),
\end{aligned}
$$

where the integration measure for $n Q$ state is given by

$$
\int[d \boldsymbol{p}]_{n}=\int d z_{1} \cdots d z_{n} \delta\left(\sum_{l=1}^{n} z_{l}-1\right) \int\left(\prod_{i=1}^{n} \frac{d^{2} \boldsymbol{p}_{i \perp}}{(2 \pi)^{2}}\right)(2 \pi)^{2} \delta^{(2)}\left(\sum_{l=1}^{n} \boldsymbol{p}_{l \perp}\right) .
$$

Here $F\left(\boldsymbol{p}_{i}\right):=F\left(z_{i}, \boldsymbol{p}_{i \perp}\right)$ denote the quark wave functions. To compute $\mathcal{N}^{(3)}(B)$, it is convenient to introduce the probability distribution $\Phi\left(z, \boldsymbol{q}_{\perp}\right)$. It also helps to compute the normalization for the $5 Q$ component and physical observables. Moreover, $\Phi\left(z, \boldsymbol{q}_{\perp}\right)$ provides information on how the valence quarks leave the longitudinal momentum fraction of the baryon and transverse momentum to the quark-antiquark pair wave function. Thus, it is given as a function of the longitudinal momentum fraction $z=q_{z} / M_{N}$ and transverse momentum $\boldsymbol{q}_{\perp}$ :

$$
\begin{aligned}
\Phi\left(z, \boldsymbol{q}_{\perp}\right)= & \int d z_{1} d z_{2} d z_{3} \delta\left(\sum_{l=1}^{3} z_{l}+z-1\right) \int\left(\prod_{i=1}^{3} \frac{d^{2} \boldsymbol{p}_{i \perp}}{(2 \pi)^{2}}\right) \\
& \times(2 \pi)^{2} \delta^{(2)}\left(\sum_{l=1}^{3} \boldsymbol{p}_{l \perp}+\boldsymbol{q}_{\perp}\right) D\left(\boldsymbol{p}_{1}, \boldsymbol{p}_{2}, \boldsymbol{p}_{3}\right),
\end{aligned}
$$



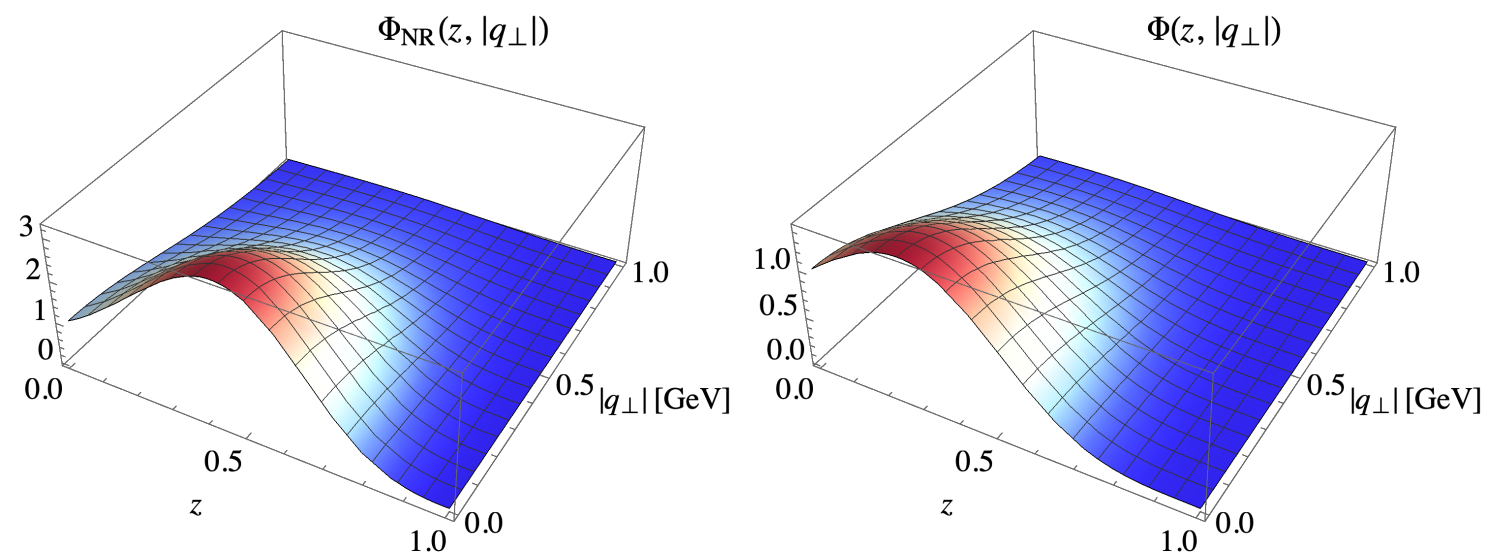

Figure 4. The left (right) panel depicts the probability distribution $\Phi\left(x, \boldsymbol{q}_{\perp}\right)$ that the valence quarks leave the longitudinal momentum fraction $z$ of the baryon momentum and transverse momentum $\boldsymbol{q}_{\perp}$ to the quark-antiquark pair wave functions in the case of the non-relativistic (relativistic) picture.

where $D\left(\boldsymbol{p}_{1}, \boldsymbol{p}_{2}, \boldsymbol{p}_{3}\right)$ is defined as

$D\left(\boldsymbol{p}_{1}, \boldsymbol{p}_{2}, \boldsymbol{p}_{3}\right):=\left[f_{\|}^{2}\left(\boldsymbol{p}_{1}\right)+p_{1 R} p_{1 L} f_{\perp}^{2}\left(\boldsymbol{p}_{1}\right)\right]\left[f_{\|}^{2}\left(\boldsymbol{p}_{2}\right)+p_{2 R} p_{2 L} f_{\perp}^{2}\left(\boldsymbol{p}_{2}\right)\right]\left[f_{\|}^{2}\left(\boldsymbol{p}_{3}\right)+p_{3 R} p_{3 L} f_{\perp}^{2}\left(\boldsymbol{p}_{3}\right)\right]$

with the discrete-level wave functions $f_{\perp, \|}\left(\boldsymbol{p}_{i}\right):=f_{\perp, \|}\left(z_{i},\left|\boldsymbol{p}_{i \perp}\right|\right)$. In the non-relativistic limit, $D\left(\boldsymbol{p}_{1}, \boldsymbol{p}_{2}, \boldsymbol{p}_{3}\right)$ is reduced to

$$
D^{\mathrm{NR}}\left(\boldsymbol{p}_{1}, \boldsymbol{p}_{2}, \boldsymbol{p}_{3}\right)=\left[f_{\|}^{\mathrm{NR}}\left(\boldsymbol{p}_{1}\right) f_{\|}^{\mathrm{NR}}\left(\boldsymbol{p}_{2}\right) f_{\|}^{\mathrm{NR}}\left(\boldsymbol{p}_{3}\right)\right]^{2} .
$$

Since in the $3 Q$ wave function there is no additional quark-antiquark pair, the normalization is thus proportional to $\Phi(0,0)$ :

$$
\mathcal{N}^{(3)}\left(B_{8}\right)=\frac{3}{2} \Phi(0,0), \quad \mathcal{N}_{1 / 2}^{(3)}\left(B_{10}\right)=\mathcal{N}_{3 / 2}^{(3)}\left(B_{10}\right)=\frac{3}{5} \Phi(0,0) .
$$

Note that the normalization of the discrete-level wave functions $f_{\perp}$ and $f_{\|}$are arbitrary. We choose it to be $\Phi(0,0)=1$. The corresponding results for the probability distribution are plotted in figure 4 for both the non-relativistic and relativistic cases.

\subsection{2 $5 Q$ Fock component}

We now compute the $5 Q$ Fock component of the baryon LCWF. Note that it has an important contribution to various baryonic observables, e.g., the axial charge $g_{A}$, the DA normalization constant $f_{N}$, and so on. Expanding eq. (4.16) to the linear order in the $Q \bar{Q}$ pair, we obtain the $5 Q$ Fock component of the baryon LCWF:

$$
\begin{aligned}
\left|\Psi^{(5) k}(B)\right\rangle= & T(B)_{j_{1} j_{2} j_{3} j_{4}, f_{5}, k}^{f_{1} f_{2} f_{3} f_{4}, j_{5}} \frac{\epsilon^{\alpha_{1} \alpha_{2} \alpha_{3}}}{\sqrt{N_{c} !}}\left[\prod_{n=1}^{N_{c}} \int \frac{d^{3} \boldsymbol{p}_{n}}{(2 \pi)^{3}} F^{j_{n} \sigma_{n}}\left(\boldsymbol{p}_{n}\right) a_{\alpha_{n} f_{n} \sigma_{n}}^{\dagger}\left(\boldsymbol{p}_{n}\right)\right] \\
& \times \int\left(\prod_{i=4}^{5} \frac{d^{3} \boldsymbol{p}_{i}}{(2 \pi)^{3}}\right) a_{\alpha_{4} f_{4} \sigma_{4}}^{\dagger}\left(\boldsymbol{p}_{\mathbf{4}}\right) \delta_{\alpha_{5}}^{\alpha_{4}} W_{j_{5} \sigma_{5}}^{j_{4} \sigma_{4}}\left(\boldsymbol{p}_{\mathbf{4}}, \boldsymbol{p}_{\mathbf{5}}\right) b^{\dagger \alpha_{5} f_{5} \sigma_{5}}\left(\boldsymbol{p}_{\mathbf{5}}\right)|0\rangle,
\end{aligned}
$$


where the indices with $1,2,3$ represent the valence quarks, whereas those with 4,5 stand for the quark and antiquark of $Q \bar{Q}$ pair, respectively. The color indices $(1,2,3)$ for the valence quarks are antisymmetric and those for the quark-antiquark pair $(4,5)$ become a color singlet. The group integral over the valence quarks and $Q \bar{Q}$-pair rotation matrices with the spin-flavor state $B_{k}^{*}$ is expressed as

$$
T(B)_{j_{1} j_{2} j_{3} j_{4}, f_{5}, k}^{f_{1} f_{2} f_{3} f_{4} j_{5}}:=\int d R B_{k}^{*}(R) R_{j_{1}}^{f_{1}} R_{j_{2}}^{f_{2}} R_{j_{3}}^{f_{3}} R_{j_{4}}^{f_{4}} R_{f_{5}}^{\dagger j_{5}}
$$

The normalization constant $\mathcal{N}^{(5)}$ is obtained by contracting the creation and annihilation operators for the valence quarks and $Q \bar{Q}$ pair. In the presence of the quark-antiquark creation operators, two additional contractions (exchange and direct contributions) are allowed. So, we get the following general expression for the normalization of the $5 Q$ component:

$$
\begin{aligned}
\mathcal{N}^{(5)}(B)= & 18 T(B)_{j_{1} j_{2} j_{3} j_{4}, f_{5}, k}^{f_{1} f_{2} f_{3} f_{4}, j_{5}} T(B)_{f_{1} f_{2} g_{3} g_{4}, j_{5}^{\prime}}^{j_{1}^{\prime} j_{2}^{\prime} j^{\prime} j_{4}^{\prime}, f_{5}, k} \int[d \boldsymbol{p}]_{5} \\
& \times F^{j_{1} \sigma_{1}}\left(\boldsymbol{p}_{1}\right) F^{j_{2} \sigma_{2}}\left(\boldsymbol{p}_{2}\right) F^{j_{3} \sigma_{3}}\left(\boldsymbol{p}_{3}\right) W_{j_{5} \sigma_{5}}^{j_{4} \sigma_{4}} F_{j_{1}^{\prime} \sigma_{1}}^{\dagger}\left(\boldsymbol{p}_{1}\right) F_{j_{2}^{\prime} \sigma_{2}}^{\dagger}\left(\boldsymbol{p}_{2}\right) \\
& \times\left[F_{j_{3}^{\prime} \sigma_{3}}^{\dagger}\left(\boldsymbol{p}_{3}\right) W_{j_{4}^{\prime} \sigma_{4}}^{\dagger j_{5}^{\prime} \sigma_{5}}\left(\boldsymbol{p}_{4}, \boldsymbol{p}_{5}\right) \delta_{f_{3}}^{g_{3}} \delta_{f_{4}}^{g_{4}}-F_{j_{3}^{\prime} \sigma_{4}}^{\dagger}\left(\boldsymbol{p}_{4}\right) W_{j_{4}^{\prime} \sigma_{3}}^{\dagger \dagger} \sigma_{5}^{\prime} \sigma_{5}\left(\boldsymbol{p}_{3}, \boldsymbol{p}_{5}\right) \delta_{f_{4}}^{g_{3}} \delta_{f_{3}}^{g_{4}}\right]
\end{aligned}
$$

with the integration measure defined in eq. (4.31). The first and second terms in the bracket are called the direct and exchange contributions, respectively. Since it was found that the exchange contributions to the normalization $\mathcal{N}^{(5)}$ are negligibly small as shown in refs. $[79,90]$, we ignore them. Note that in the case of the direct contributions the $Q \bar{Q}$-pair momenta are almost decoupled from the valence quark momenta. Thus, we can define the following function with the valence quarks and $Q \bar{Q}$ pair factorized:

$$
K_{J}:=\frac{M^{2}}{2 \pi} \int \frac{d^{3} \boldsymbol{q}}{(2 \pi)^{3}} \Phi\left(\frac{q_{z}}{M_{N}}, \boldsymbol{q}_{\perp}\right) \theta\left(q_{z}\right) q_{z} G_{J}\left(q_{z}, \boldsymbol{q}_{\perp}\right),
$$

where $G_{J}$ are quark-antiquark probability distributions with $J=\pi \pi, \sigma \sigma$, and 33, which arises from the quark-antiquark loops:

$$
\begin{aligned}
& G_{\sigma \sigma}\left(q_{z}, \boldsymbol{q}_{\perp}\right)=\Sigma^{2}(\boldsymbol{q}) \int_{0}^{1} d y \int \frac{d^{2} \mathcal{Q}_{\perp}}{(2 \pi)^{2}}\left[\frac{M^{2}(2 y-1)^{2}+\mathcal{Q}_{\perp}^{2}}{\left(\mathcal{Q}_{\perp}^{2}+M^{2}+y(1-y) \boldsymbol{q}^{2}\right)^{2}}-\left(M-M_{\mathrm{PV}}\right)\right] \\
& G_{\pi \pi}\left(q_{z}, \boldsymbol{q}_{\perp}\right)=\Pi^{2}(\boldsymbol{q}) \int_{0}^{1} d y \int \frac{d^{2} \mathcal{Q}_{\perp}}{(2 \pi)^{2}}\left[\frac{M^{2}+\mathcal{Q}_{\perp}^{2}}{\left(\mathcal{Q}_{\perp}^{2}+M^{2}+y(1-y) \boldsymbol{q}^{2}\right)^{2}}-\left(M-M_{\mathrm{PV}}\right)\right] \\
& G_{33}\left(q_{z}, \boldsymbol{q}_{\perp}\right)=\frac{q_{z}^{2}}{\boldsymbol{q}^{2}} \Pi^{2}(\boldsymbol{q}) \int_{0}^{1} d y \int \frac{d^{2} \mathcal{Q}_{\perp}}{(2 \pi)^{2}}\left[\frac{M^{2}+\mathcal{Q}_{\perp}^{2}}{\left(\mathcal{Q}_{\perp}^{2}+M^{2}+y(1-y) \boldsymbol{q}^{2}\right)^{2}}-\left(M-M_{\mathrm{PV}}\right)\right]
\end{aligned}
$$

Since $G_{J}$ are divergent logarithmically, we introduce the Pauli-Villars regulator to tame them. After some straightforward manipulations, one arrives at the baryon normalization 
constant $\mathcal{N}^{(5)}$ expressed in terms of $K_{J}$

$$
\begin{aligned}
\mathcal{N}^{(5)}\left(B_{8}\right) & =\frac{3}{10}\left(11 K_{\pi \pi}+23 K_{\sigma \sigma}\right) \\
\mathcal{N}_{1 / 2}^{(5)}\left(B_{10}\right) & =\frac{3}{20}\left(11 K_{\pi \pi}+6 K_{33}+17 K_{\sigma \sigma}\right) \\
\mathcal{N}_{3 / 2}^{(5)}\left(B_{10}\right) & =\frac{3}{20}\left(15 K_{\pi \pi}-6 K_{33}+17 K_{\sigma \sigma}\right)
\end{aligned}
$$

\subsection{Distribution amplitudes}

So far, we have defined the baryon LCWF normalized to be unity in the instant form for simplicity. However, it is more convenient to introduce the LCWF given as a function of the light-cone variables, since it can be directly applied to many baryonic observables such as the electromagnetic form factors of the nucleon $[2,3]$. Thus, we reexpress it in the covariantly normalized form in terms of the light-cone variables, keeping the physics intact:

$$
\begin{aligned}
\left|N\left(p_{N}, \lambda\right)\right\rangle= & c_{0}^{\mathcal{R}, J_{z}} T(B)_{j_{1} j_{2} j_{3}, k}^{f g h} \int\left[\frac{d x}{\sqrt{x}}\right]_{3} \\
& \times \int\left[d k_{\perp}\right]_{3} F^{j_{1} \sigma_{1}}\left(\boldsymbol{k}_{1}\right) F^{j_{2} \sigma_{2}}\left(\boldsymbol{k}_{2}\right) F^{j_{3} \sigma_{3}}\left(\boldsymbol{k}_{3}\right)\left|f^{\sigma_{1}} g^{\sigma_{2}} h^{\sigma_{3}}\right\rangle,
\end{aligned}
$$

where the fermion states are normalized to be eqs. (2.9) and (2.10) and the normalization of the LCWF is expressed as

$$
c_{0}^{\mathcal{R}, J_{z}}=\sqrt{\frac{(4 \pi)^{2}}{\mathcal{N}^{(3)}+\mathcal{N}^{(5)} \ldots}} .
$$

The well-known abbreviated notations of the integration measures are adopted as follows:

$$
\begin{aligned}
\int\left[\frac{d x}{\sqrt{x}}\right]_{n}:=\int\left[\prod_{j=1}^{n} \frac{d x_{j}}{\sqrt{x_{j}}}\right] \delta\left(1-\sum_{l=1}^{n} x_{l}\right), \\
\int\left[d k_{\perp}\right]_{n}:=\int\left[\prod_{j=1}^{n} \frac{d \boldsymbol{k}_{j \perp}}{2(2 \pi)^{3}}\right] 2(2 \pi)^{3} \delta^{(2)}\left(\sum_{l=1}^{n} \boldsymbol{k}_{l \perp}\right) .
\end{aligned}
$$

Applying the three quark field operators to the baryon LCWF (4.42) in eq. (2.3), one can extract respectively the DAs for the nucleon and $\Delta$ baryon

$$
\begin{aligned}
f_{N} \varphi_{N} & =24 \sqrt{6} c_{0}^{\mathbf{8}, 1 / 2} \int\left[d k_{\perp}\right] T_{j_{1} j_{2} j_{3}, 1}^{12} F^{j_{1} 1}\left(\boldsymbol{k}_{1}\right) F^{j_{2} 2}\left(\boldsymbol{k}_{2}\right) F^{j_{3} 1}\left(\boldsymbol{k}_{3}\right), \\
f_{\Delta}^{1 / 2} \varphi_{\Delta}^{1 / 2} & =-24 \sqrt{6} c_{0}^{\mathbf{1 0}, 1 / 2} \int\left[d k_{\perp}\right] T_{j_{1} j_{2} j_{3}, 1}^{111} F^{j_{1} 1}\left(\boldsymbol{k}_{1}\right) F^{j_{2} 2}\left(\boldsymbol{k}_{2}\right) F^{j_{3} 1}\left(\boldsymbol{k}_{3}\right), \\
f_{\Delta}^{3 / 2} \varphi_{\Delta}^{3 / 2} & =-24 \sqrt{3} c_{0}^{\mathbf{1 0}, 3 / 2} \int\left[d k_{\perp}\right] T_{j_{1} j_{2} j_{3}, 1}^{111} F^{j_{1} 1}\left(\boldsymbol{k}_{1}\right) F^{j_{2} 1}\left(\boldsymbol{k}_{2}\right) F^{j_{3} 1}\left(\boldsymbol{k}_{3}\right) .
\end{aligned}
$$


Summing over the isospin indices, we obtain the explicit expressions for the DAs

$$
\begin{aligned}
& f_{N} \varphi_{N}=4 \sqrt{3} c_{0}^{8,1 / 2} \int\left[d k_{\perp}\right]\left[f_{\|}\left(\boldsymbol{k}_{\mathbf{1}}\right) f_{\|}\left(\boldsymbol{k}_{\mathbf{2}}\right) f_{\|}\left(\boldsymbol{k}_{\mathbf{3}}\right)\right. \\
& \left.-k_{1 L} k_{2 R} f_{\perp}\left(\boldsymbol{k}_{\mathbf{1}}\right) f_{\perp}\left(\boldsymbol{k}_{\mathbf{2}}\right) f_{\|}\left(\boldsymbol{k}_{\mathbf{3}}\right)+2 k_{3 L} k_{2 R} f_{\perp}\left(\boldsymbol{k}_{\mathbf{3}}\right) f_{\perp}\left(\boldsymbol{k}_{\mathbf{2}}\right) f_{\|}\left(\boldsymbol{k}_{\mathbf{1}}\right)\right], \\
& f_{\Delta}^{1 / 2} \varphi_{\Delta}^{1 / 2}=-\frac{24}{\sqrt{5}} c_{0}^{\mathbf{1 0 , 1 / 2}} \int\left[d k_{\perp}\right]\left[f_{\|}\left(\boldsymbol{k}_{\mathbf{1}}\right) f_{\|}\left(\boldsymbol{k}_{\mathbf{2}}\right) f_{\|}\left(\boldsymbol{k}_{\mathbf{3}}\right)\right. \\
& \left.-k_{1 L} k_{2 R} f_{\perp}\left(\boldsymbol{k}_{\mathbf{1}}\right) f_{\perp}\left(\boldsymbol{k}_{\mathbf{2}}\right) f_{\|}\left(\boldsymbol{k}_{\mathbf{3}}\right)-k_{3 L} k_{2 R} f_{\perp}\left(\boldsymbol{k}_{\mathbf{3}}\right) f_{\perp}\left(\boldsymbol{k}_{\mathbf{2}}\right) f_{\|}\left(\boldsymbol{k}_{\mathbf{1}}\right)\right], \\
& f_{\Delta}^{3 / 2} \varphi_{\Delta}^{3 / 2}=12 \sqrt{\frac{6}{5}} c_{0}^{\mathbf{1 0}, 3 / 2} \int\left[d k_{\perp}\right]\left[f_{\|}\left(\boldsymbol{k}_{\mathbf{1}}\right) f_{\|}\left(\boldsymbol{k}_{\mathbf{2}}\right) f_{\|}\left(\boldsymbol{k}_{\mathbf{3}}\right)\right] .
\end{aligned}
$$

If the LCWF is normalized to the $3 Q$ components in the non-relativistic limit, then the normalization constants $f_{N}^{(3)}, f_{\Delta}^{1 / 2,(3)}, f_{\Delta}^{3 / 2,(3)}$ become

$$
\left|\frac{f_{\Delta}^{1 / 2,(3)}}{f_{N}^{(3)}}\right|=\sqrt{6}, \quad\left|\frac{f_{\Delta}^{3 / 2,(3)}}{f_{N}^{(3)}}\right|=3, \quad\left|\frac{f_{\Delta}^{1 / 2,(3)}}{f_{\Delta}^{3 / 2,(3)}}\right|=\sqrt{\frac{2}{3}} .
$$

The expressions for the distribution amplitudes $A, V$ and $T$ are listed in appendix B.

\section{Numerical results and discussion}

To compute the baryon LCWFs, we have to fix the parameters of the $\chi$ QSM. The PauliVillars cutoff mass is fixed to be $M_{\mathrm{PV}}=557 \mathrm{MeV}$ by reproducing the pion decay constant $F_{\pi}=93 \mathrm{MeV}$. As mentioned previously, the dynamical quark mass $M$ is originally momentum-dependent, which comes from the instanton zero mode. Using the standard values for the instanton ensemble, i.e. the instanton density $N / V=(1 \mathrm{fm})^{-1}$ and the average size of the instanton $\rho=\frac{1}{3} \mathrm{fm}$, one obtains $M=M(0)=345 \mathrm{MeV}$ at the zero quark virtuality. We will use this value in this work. By putting all the obtained functions into eq. (4.39), the numerical results for $K_{\pi \pi}, K_{\sigma \sigma}$ and $K_{33}$ are evaluated to be

$$
\begin{aligned}
& K_{\sigma \sigma}^{\mathrm{NR}}=0.059, \quad K_{\pi \pi}^{\mathrm{NR}}=0.128, \quad K_{33}^{\mathrm{NR}}=0.077, \\
& K_{\sigma \sigma}=0.029, \quad K_{\pi \pi}=0.075, \quad K_{33}=0.041 .
\end{aligned}
$$

Employing these results, we determine the non-relativistic and relativistic normalizations for both the baryon octet and decuplet

$$
\mathcal{N}^{(5)}\left(B_{8}\right)=0.45, \quad \mathcal{N}_{1 / 2}^{(5)}\left(B_{10}\right)=0.23, \quad \mathcal{N}_{3 / 2}^{(5)}\left(B_{10}\right)=0.21 .
$$

We list in table 1 each contribution of the $3 Q$ and $5 Q$ components respectively to the normalization constant for the baryon octet and decuplet in the nonrelativistic case. In table 2, we list them in the relativistic case. As shown in tables 1 and 2, the contribution from the $5 Q$ Fock component to the normalization constant is sizable. When we consider the relativistic case, the fractions of the $5 Q$ Fock component are reduced by about $10 \%$. 


\begin{tabular}{c|cc}
\hline$B_{\mathcal{R}, J_{z}}$ & $3 Q \equiv \frac{\mathcal{N}_{J_{z}}^{(3)}\left(B_{\mathcal{R}}\right)}{\mathcal{N}_{J_{z}}^{(3)}\left(B_{\mathcal{R}}\right)+\mathcal{N}_{J_{z}}^{(5)}\left(B_{\mathcal{R}}\right)}$ & $5 Q \equiv \frac{\mathcal{N}_{J_{z}}^{(5)}\left(B_{\mathcal{R}}\right)}{\mathcal{N}_{J_{z}}^{(3)}\left(B_{\mathcal{R}}\right)+\mathcal{N}_{J_{z}}^{(5)}\left(B_{\mathcal{R}}\right)}$ \\
\hline$B_{8,1 / 2}$ & $64 \%$ & $36 \%$ \\
$B_{10,1 / 2}$ & $58 \%$ & $42 \%$ \\
$B_{10,3 / 2}$ & $62 \%$ & $38 \%$ \\
\hline
\end{tabular}

Table 1. $3 Q$ and $5 Q$ fractions of the normalization constants for the baryon octet and decuplet in the nonrelativistic case. $\mathcal{R}$ stands for the $\mathrm{SU}(3)$ representation of the baryon multiplet. The spin projection of the corresponding baryon multiplet is denoted by $J_{z}$.

\begin{tabular}{c|cc}
\hline$B_{\mathcal{R}, J_{z}}$ & $3 Q \equiv \frac{\mathcal{N}_{J_{z}}^{(3)}\left(B_{\mathcal{R}}\right)}{\mathcal{N}_{J_{z}}^{(3)}\left(B_{\mathcal{R}}\right)+\mathcal{N}_{J_{z}}^{(5)}\left(B_{\mathcal{R}}\right)}$ & $5 Q \equiv \frac{\mathcal{N}_{J_{z}}^{(5)}\left(B_{\mathcal{R}}\right)}{\mathcal{N}_{J_{z}}^{(3)}\left(B_{\mathcal{R}}\right)+\mathcal{N}_{J_{z}}^{(5)}\left(B_{\mathcal{R}}\right)}$ \\
\hline$B_{8,1 / 2}$ & $77 \%$ & $23 \%$ \\
$B_{10,1 / 2}$ & $72 \%$ & $28 \%$ \\
$B_{10,3 / 2}$ & $74 \%$ & $26 \%$ \\
\hline
\end{tabular}

Table 2. $3 Q$ and $5 Q$ fractions of the normalization constants for the baryon octet and decuplet in the relativistic case. $\mathcal{R}$ stands for the $\mathrm{SU}(3)$ representation of the baryon multiplet. The spin projection of the corresponding baryon multiplet is denoted by $J_{z}$.
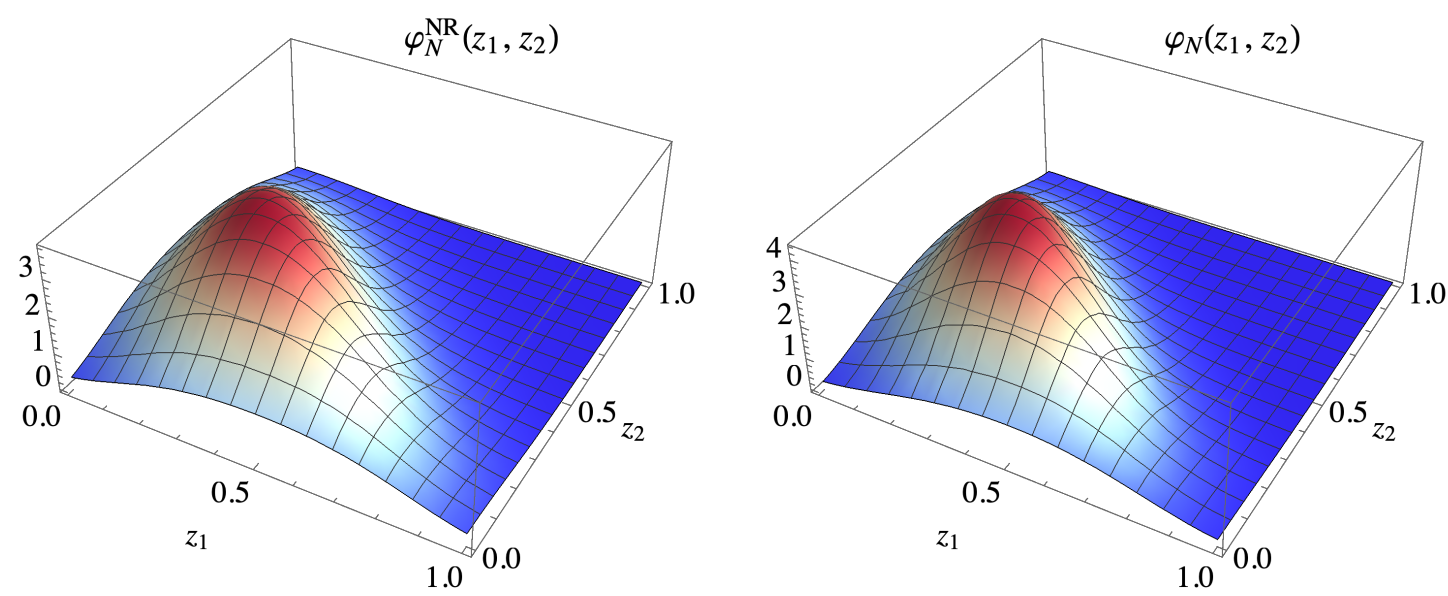

Figure 5. Nucleon distribution amplitudes in the non-relativistic (left panel) and relativistic (right panel) cases, given as functions of $z_{1}$ and $z_{2}$.

This indicates that it is crucial to consider both the $5 Q$ Fock component and the relativistic corrections in the baryon LCWFs. The normalization constants of the $3 Q$ and $5 Q$ Fock components have significant physical implications: the amount of each component directly yields information on how much the quarks inside a baryon carry the fraction of the baryon longitudinal momentum. For example, the $3 Q$ quarks inside the nucleon carry $77 \%$ of the nucleon momentum in the relativistic case. 
In figure 5, we draw the numerical results for the nucleon DAs $\varphi_{N}\left(z_{1}, z_{2}\right) \equiv$ $\varphi_{N}\left(z_{1}, z_{2}, 1-z_{1}-z_{2}\right)$ in both the nonrelativistic (left panel) and relativistic (right panel) cases. The decomposed results for $F\left(z_{1}, z_{2}\right)$ are presented in appendix B. As shown in eq. (2.6), the $\varphi_{N}$ consists of both the symmetric $V\left(z_{1}, z_{2}, z_{3}\right)$ and antisymmetric $A\left(z_{1}, z_{2}, z_{3}\right)$ parts under the exchange $z_{1} \leftrightarrow z_{2}$. From eq. (4.46), we see that the antisymmetric part of the nucleon DA originates from the relativistic corrections, which implies that the nucleon $\mathrm{DA} \varphi_{N}^{\mathrm{NR}}\left(z_{1}, z_{2}\right)$ becomes symmetric in the nonrelativistic limit when the exchange $z_{1} \leftrightarrow z_{2}$ is considered, i.e., $A\left(z_{1}, z_{2}\right)=0$. On the other hand, the antisymmetric DA $A\left(z_{1}, z_{2}\right)$ comes into play in the relativistic case, so that the configuration of the nucleon DA is distorted from the symmetric shape. However, since the antisymmetric DA contributes only weakly to the nucleon DA (less than $5 \%$ ), $\varphi_{N}$ turns out almost symmetric. Interestingly, these results are rather close to that of the asymptotic nucleon DA, $\varphi_{N}^{\text {asy }}=120 z_{1} z_{2} z_{3}$. The present results are consistent with those from the lattice QCD [43-48]. Note that the QCD sum rules $[22,24,26,27]$ give rather asymmetric forms of the nucleon DA.

We want to stress that the present model is valid only in the region $z N_{c} \sim 1$. This means that the approximation used here brings about the non-zero values of the DAs at the endpoints $z=0$ and $z=1$. Since at $z=0$ the virtuality of the partons inside a nucleon becomes very large, the constant dynamical quark mass is no longer plausible. Thus, it is inevitable to use the momentum-dependent dynamical quark mass $M(p)$ to explain the endpoint behavior of the baryon DAs. As pointed out in ref. [66], the baryon mass is very large in the large $N_{c}$ limit $\left(M_{B} \sim N_{c}\right)$, so that the recoil effects of the baryon are neglected. This implies that the baryon DAs may not vanish at $z=1$. Moreover, when the partons are very virtual, the gluons start to radiate and will affect the DAs considerably, the Sudakov form factors should be included in the DAs. These were neglected here [72, 74]. One faces the same problem in the calculation of the structure functions $[66,68]$.

In figure 6 , we draw the results for the $\Delta$ baryon DAs with the spin projection $1 / 2$ and $3 / 2$ in non-relativistic and relativistic cases. The main features are similar to those of the nucleon shown in figure 5 . The $\Delta$ DAs $\varphi_{\Delta}^{1 / 2, \mathrm{NR}}, \varphi_{\Delta}^{3 / 2, \mathrm{NR}}$ become naturally identical to that $\varphi_{N}^{\mathrm{NR}}$ of the nucleon. The present results for the $\Delta$ baryon DAs are also close to the asymptotic DA and are consistent with those from the QCD sum rules [35].

The baryon decay constants or the DA normalization constants, $f_{N}, f_{\Delta}^{1 / 2}$ and $f_{\Delta}^{3 / 2}$, contain essential information on the baryon DAs, which were defined in eqs. (2.3) and (3.1). We list in table 3 the numerical results for both the $3 Q$ and $3 Q+5 Q$ contributions to them in the nonrelativistic and relativistic cases. Interestingly, in the nonrelativistic limit, we have derived the relations between the DA normalization constants for the nucleon and $\Delta$ baryon given in eq. (4.47). The numerical results listed in table 3 satisfy these relations. Moreover, we find that both the $5 Q$ component and the relativistic corrections are significant to determine the DA normalization constants.

These normalization constants are scale-dependent as mentioned already. The scale of the present model can be related to the average size of the instanton, of which the value is $\rho \sim 0.33 \mathrm{fm}$. So, we will approximately take $\mu_{0}^{2} \sim 0.36 \mathrm{GeV}^{2}$ as the scale of the $\chi$ QSM $[85,91,92]$. To compare the present results with those from other works, one has to match the scale by using the renormalization group equation for the DA normalization 

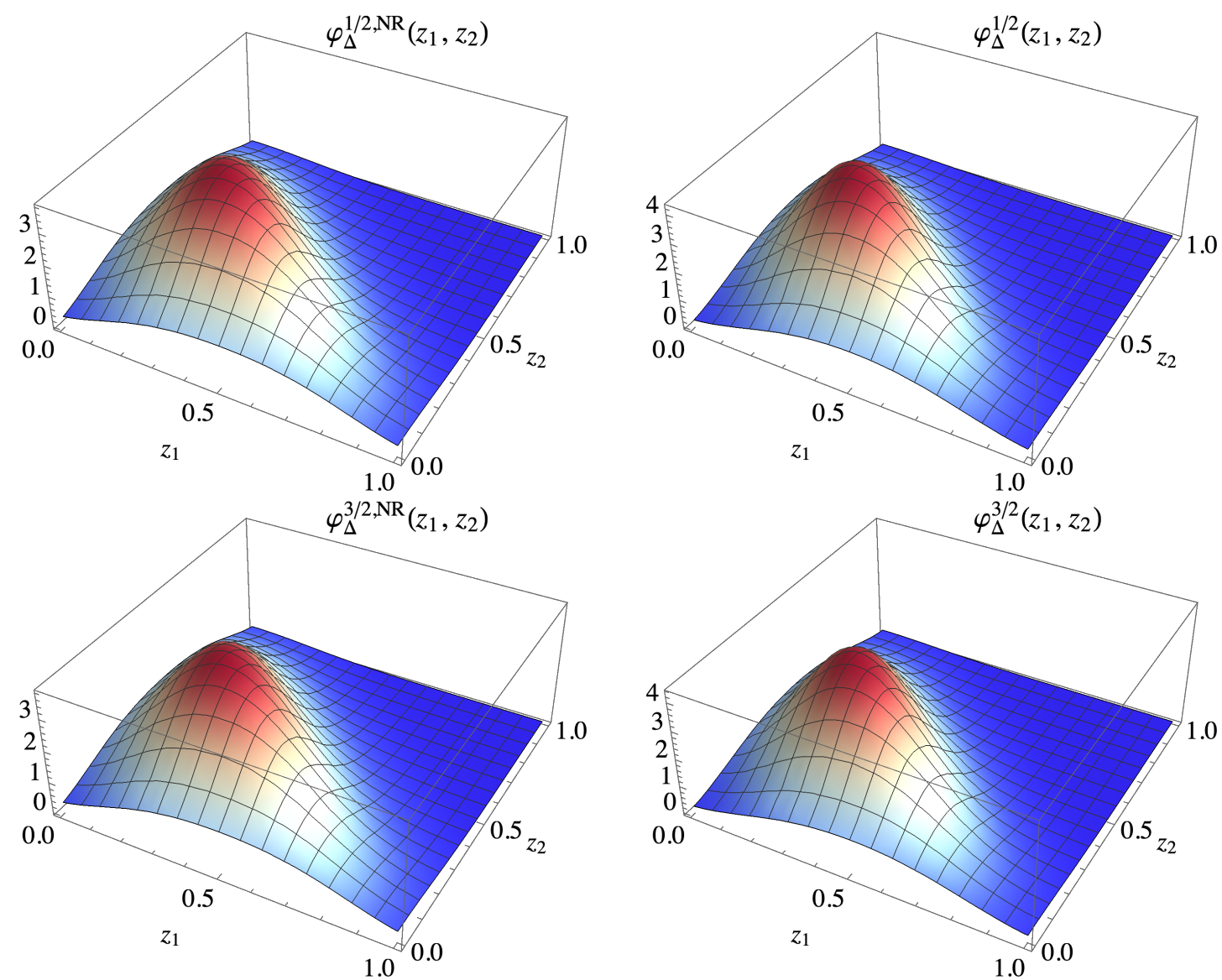

Figure 6. $\Delta$ distribution amplitudes in the non-relativistic (left panel) and relativistic (right panel) cases.

\begin{tabular}{c|cccc}
\hline & \multicolumn{2}{|c}{ Non-relativistic } & \multicolumn{2}{c}{ Relativistic } \\
\hline$f_{B}$ & $3 Q$ & $3 Q+5 Q$ & $3 Q$ & $3 Q+5 Q$ \\
\hline$\left|f_{N}\right|$ & 6.2 & 5.0 & 4.8 & 4.2 \\
$\left|f_{\Delta}^{1 / 2}\right|$ & 15.2 & 11.6 & 14.9 & 12.7 \\
$\left|f_{\Delta}^{3 / 2}\right|$ & 18.7 & 14.7 & 15.5 & 13.4 \\
\hline
\end{tabular}

Table 3. Non-relativistic and relativisic DA normalizations of the nucleon and $\Delta$ baryon in units of $10^{-3} \mathrm{GeV}^{2}$. 
constants $f_{N}, f_{\Delta}^{1 / 2}$ and $f_{\Delta}^{3 / 2}$. The one-loop evolution of them is derived in refs. $[2,36]$

$$
f_{B}\left(\mu^{2}\right)=f_{B}\left(\mu_{0}^{2}\right)\left(\frac{\alpha_{\mathrm{s}}\left(\mu^{2}\right)}{\alpha_{\mathrm{s}}\left(\mu_{0}^{2}\right)}\right)^{\gamma_{0}},
$$

where the anomalous dimension $\gamma_{0}=2 /\left(3 \beta_{0}\right)$ is given by refs. $[2,93,94]$ with the beta function $\beta_{0}=11-2 n_{f} / 3$ for the strong coupling constant $\alpha_{\mathrm{s}}\left(\mu^{2}\right)$. Being evolved to a high normalization point, for example, $\mu=2 \mathrm{GeV}$, the DA normalization constants are respectively found to be

$$
\left|f_{N}\right|=3.9 \times 10^{-3} \mathrm{GeV}^{2}, \quad\left|f_{\Delta}^{1 / 2}\right|=11.7 \times 10^{-3} \mathrm{GeV}^{2}, \quad\left|f_{\Delta}^{3 / 2}\right|=12.3 \times 10^{-3} \mathrm{GeV}^{2} .
$$

Though we take $\mu_{0}^{2} \sim 0.36 \mathrm{GeV}^{2}$ as the scale of the present model, this is not uniquely determined. In fact, it depends on a parameter varied in the variational estimate of bulk properties of the instanton medium. In refs. [85, 91, 92] the scale of the $\chi$ QSM was estimated to be $\mu_{0}=(0.6-1.4) \mathrm{GeV}$ with the parameter varied. Owing to the weak dependence of the DA normalization constants on the scale evolution, the corresponding error lies within around $5 \%$. So, $\mu_{0}^{2}=0.36 \mathrm{GeV}^{2}$ is a reasonable choice.

In refs. [79, 81, 90], the DA normalization constant for the nucleon from the $7 Q$ contribution $\mathcal{N}^{(7)}$ was investigated. Though we do not evaluate them explicitly, we use the value obtained in refs. $[79,81,90]$ and estimate $f_{N}$ at $\mu=2 \mathrm{GeV}$ :

$$
\left|f_{N}\right| \simeq 3.7 \times 10^{-3} \mathrm{GeV}^{2} \quad(3 Q+5 Q+7 Q) .
$$

We then expect that further higher Fock contributions are suppressed. This value was first introduced and estimated in the QCD sum rules $[22,24,26,27,35]$, where $\left|f_{N}\right|$ was estimated to be $\left|f_{N}(\mu \simeq 1 \mathrm{GeV})\right| \simeq 5.0 \times 10^{-3} \mathrm{GeV}^{2}$ [35]. This value is rather large compared with the present result. Recently, however, it was obtained from the lattice QCD [48] as follows: $\left|f_{N}(\mu=2 \mathrm{GeV})\right|=3.54_{-4}^{+6} \times 10^{-3} \mathrm{GeV}^{2}$. The present result given in eq. (5.5) is in good agreement with this lattice data. For the $\Delta$ baryon, the QCD sum rules [35] yield the following results: $\left|f_{\Delta}^{1 / 2}(\mu \simeq 1 \mathrm{GeV})\right|=(12 \pm 0.2) \times 10^{-3} \mathrm{GeV}^{2}$ and $\left|f_{\Delta}^{3 / 2}(\mu \simeq 1 \mathrm{GeV})\right|=14 \times 10^{-3} \mathrm{GeV}^{2}$, which are consistent with the present ones.

\section{Summary and conclusions}

In the present work, we aimed at providing the leading-twist distribution amplitudes of the nucleon and $\Delta$ baryon and their normalization constants $f_{N}, f_{\Delta}^{1 / 2}$ and $f_{\Delta}^{3 / 2}$ within the chiral quark-soliton model. We first defined the distribution amplitudes by means of the vacuum-to-baryon matrix elements of trilocal QCD operators. The leading-twist distribution amplitudes are related to the light-cone wave functions with the orbital angular momentum $L_{z}=0$ involved in the valence three-quark Fock component. In the chiral quark-soliton model, the explicit light-cone wave functions of the baryon octet and decuplet are derived, which consist of the valence quark and quark-antiquark (pair) wave functions. Expanding the vacuum wave function or the Dirac sea, we were able to construct the light-cone wave function for the higher Fock components, i.e, 3, 5, 7...-wave functions. As 
pointed out in refs. [78, 81], it turned out that the five-quark contributions significantly contribute to the normalization for the baryon light-cone wave functions and are found to be $\sim 15 \%$. Having included the five-quark contributions, we estimated the distribution amplitudes of the nucleon and $\Delta$ baryon. The nucleon distribution amplitude $\varphi_{N}$ is found to be almost symmetric under the exchange of the first two arguments, which is consistent with the recent results from the lattice QCD [46, 48]. Interestingly, the normalization constants for the proton distribution amplitude are obtained to be $\left|f_{N}(\mu=2 \mathrm{GeV})\right|=3.7 \times$ $10^{-3} \mathrm{GeV}^{2}$, which is very close to the lattice data, $f_{N}(\mu \sim 2 \mathrm{GeV})=3.54 \times 10^{-3} \mathrm{GeV}^{2}[48]$. The distribution amplitudes of the $\Delta$ baryon $\varphi_{\Delta}^{1 / 2}$ and $\varphi_{\Delta}^{3 / 2}$ are respectively found to be almost and totally symmetric, and their normalization constants are respectively obtained to be $\left|f_{\Delta}^{1 / 2}(\mu=2 \mathrm{GeV})\right|=11.7 \times 10^{-3} \mathrm{GeV}^{2}$ and $\left|f_{\Delta}^{3 / 2}(\mu=2 \mathrm{GeV})\right|=12.3 \times 10^{-3} \mathrm{GeV}^{2}$. We anticipate that the results from the lattice QCD or several theoretical approaches for the $\Delta$ baryon distribution amplitudes will soon come out.

The nucleon and $\Delta$ baryon light-cone wave functions obtained from the present work can be applied to the study of the electromagnetic and mechanical properties of the nucleon and $\Delta$. Since the electromagnetic and gravitational form factors can be directly derived from the light-cone wave functions, we can immediately get access to the transverse charge densities for the nucleon and $\Delta$ baryon. More importantly, the mechanical properties of both the nucleon and $\Delta$ with the stability conditions can be scrutinized by using the present results, since they provide essential information on how the nucleon [95-99] and $\Delta$ [100-102] are shaped physically. On the other hand, it is also of great importance to investigate the baryon light-cone wave functions with the momentum-dependent dynamical quark mass obtained from the instanton vacuum. This will make the baryon light-cone wave functions to satisfy the correct endpoint behavior. Recently, the chiral quark-soliton model or the pion mean-field approach has been successfully extended to the description of singly heavy baryons [103-115]. Thus, it is of great interest to study the light-cone wave functions of the singly heavy baryons. The corresponding studies are under way.

\section{Acknowledgments}

The present work was supported by Basic Science Research Program through the National Research Foundation of Korea funded by the Korean government (Ministry of Education, Science and Technology, MEST), Grant-No. 2021R1A2C2093368 and 2018R1A5A1025563. J.-Y.K is supported by the Deutscher Akademischer Austauschdienst(DAAD) doctoral scholarship.

\section{A Baryon rotational wave functions}

The wave functions for the baryon octet are generically expressed as mixed tensors, $P_{f}^{g}$, whereas those for the baryon decuplet as symmetric ones, $D_{f_{1} f_{2} f_{3}}$ with three quark indices. This means that the Wigner $D$ functions for the baryon octet and decuplet can be expressed 
as follows:

$$
\begin{aligned}
{\left[D^{(8,1 / 2) *}(R)\right]_{f, k}^{g} } & \sim \epsilon_{k l} R_{f}^{\dagger l} R_{3}^{g}, \\
{\left[D^{(10,3 / 2) *}(R)\right]_{\left\{f_{1} f_{2} f_{3}\right\},\left\{k_{1} k_{2} k_{3}\right\}}^{g} } & \left.\sim \epsilon_{k_{1}^{\prime} k_{1}} \epsilon_{k_{2}^{\prime} k_{2}} \epsilon_{k_{3}^{\prime} k_{3}} R_{f_{1}}^{\dagger k_{1}^{\prime}} R_{f_{2}}^{\dagger k_{2}^{\prime}} R_{f_{3}}^{\dagger k_{3}^{\prime}}\right|_{\left\{f_{1} f_{2} f_{3}\right\}},
\end{aligned}
$$

where $\epsilon_{k l}$ stands for the antisymmetric tensor. The $k=1$ means the spin-up baryon state, whereas $k=2$ represents the spin-down state. The wave functions for the baryon decuplet are fully symmetrized in the flavor $\left\{f_{1} f_{2} f_{3}\right\}$ and spin projection $\left\{k_{1} k_{2} k_{3}\right\}$ indices. The flavor part of the baryon octet are explicitly written as [116]:

$$
\begin{array}{llrl}
P_{1}^{3} & =N^{+}, & P_{2}^{3}=N^{0}, & P_{1}^{2}=\Sigma^{+}, \quad P_{2}^{1}=\Sigma^{-}, \\
P_{1}^{1}=\frac{1}{\sqrt{2}} \Sigma^{0}+\frac{1}{\sqrt{6}} \Lambda^{0}, & P_{2}^{2}=-\frac{1}{\sqrt{2}} \Sigma^{0}+\frac{1}{\sqrt{6}} \Lambda^{0}, & \\
P_{3}^{3}=-\sqrt{\frac{2}{3}} \Lambda^{0}, & P_{3}^{2}=\Xi^{0}, & P_{3}^{1}=-\Xi^{-},
\end{array}
$$

and that of the decuplet $D_{\left\{f_{1} f_{2} f_{3}\right\}}$ are given as

$$
\begin{array}{llll}
D_{111}=\sqrt{6} \Delta^{++}, & D_{112}=\sqrt{2} \Delta^{+}, & D_{122}=\sqrt{2} \Delta^{0}, & D_{222}=\sqrt{6} \Delta^{-}, \\
D_{113}=\sqrt{2} \Sigma^{*+}, & D_{123}=-\Sigma^{* 0}, & D_{223}=-\sqrt{2} \Sigma^{*-}, & D_{133}=\sqrt{2} \Xi^{* 0}, \\
D_{233}=\sqrt{2} \Xi^{*-}, & D_{333}=-\sqrt{6} \Omega^{-} . & &
\end{array}
$$

The prefactors for the wave functions are determined by normalizing the rotational wave functions

$$
\int d R B_{\mathrm{spin}}^{*}(R) B^{\mathrm{spin}}(R)=1 .
$$

For example, the nucleon state is explcitly expressed as

$$
N_{k}^{+*}(R)=\sqrt{8} \epsilon_{k l} R_{1}^{\dagger l} R_{3}^{3}, \quad N_{k}^{0 *}(R)=\sqrt{8} \epsilon_{k l} R_{2}^{\dagger l} R_{3}^{3},
$$

whereas the $\Delta$ baryon with spin projection $3 / 2(\uparrow \uparrow \uparrow)$ and $1 / 2(\uparrow)$ are written as

$$
\begin{array}{ll}
\Delta_{\uparrow \uparrow \uparrow}^{++*}(R)=\sqrt{10} R_{1}^{\dagger 2} R_{1}^{\dagger 2} R_{1}^{\dagger 2}, & \Delta_{\uparrow \uparrow \uparrow}^{+*}(R)=\sqrt{30} R_{1}^{\dagger 2} R_{1}^{\dagger 2} R_{2}^{\dagger 2}, \\
\Delta_{\uparrow}^{++*}(R)=\sqrt{30} R_{1}^{\dagger 2} R_{1}^{\dagger 2} R_{1}^{\dagger 1}, & \Delta_{\uparrow}^{+*}(R)=\sqrt{10}\left(R_{1}^{\dagger 2} R_{1}^{\dagger 2} R_{2}^{\dagger 1}+2 R_{2}^{\dagger 2} R_{1}^{\dagger 2} R_{1}^{\dagger 1}\right) .
\end{array}
$$

For different spin projections, the integral becomes zero. The rotational wave functions belonging to different baryons satisfy the orthogonality. 


\section{B Distribution amplitudes}

The distribution amplitudes are listed for the nucleon

$$
\begin{array}{r}
f_{N} T_{N}=-12 \sqrt{6} c_{0}^{8,1 / 2} \int\left[d k_{\perp}\right] T_{j_{1} j_{2} j_{3}, 1}^{112} F^{j_{1} 1}\left(\boldsymbol{p}_{1}\right) F^{j_{2} 1}\left(\boldsymbol{p}_{2}\right) F^{j_{3} 2}\left(\boldsymbol{p}_{3}\right), \\
f_{N} V_{N}=12 \sqrt{6} c_{0}^{\mathbf{8}, 1 / 2} \int\left[d k_{\perp}\right] T_{j_{1} j_{2} j_{3}, 1}^{112}\left[F^{j_{1} 2}\left(\boldsymbol{p}_{1}\right) F^{j_{2} 1}\left(\boldsymbol{p}_{2}\right) F^{j_{3} 1}\left(\boldsymbol{p}_{3}\right)\right. \\
\left.+F^{j_{1} 1}\left(\boldsymbol{p}_{1}\right) F^{j_{2} 2}\left(\boldsymbol{p}_{2}\right) F^{j_{3} 1}\left(\boldsymbol{p}_{3}\right)\right], \\
f_{N} A_{N}=12 \sqrt{6} c_{0}^{\mathbf{8}, 1 / 2} \int\left[d k_{\perp}\right] T_{j_{1} j_{2} j_{3}, 1}^{112}\left[F^{j_{1} 2}\left(\boldsymbol{p}_{1}\right) F^{j_{2} 1}\left(\boldsymbol{p}_{2}\right) F^{j_{3} 1}\left(\boldsymbol{p}_{3}\right)\right. \\
\left.-F^{j_{1} 1}\left(\boldsymbol{p}_{1}\right) F^{j_{2} 2}\left(\boldsymbol{p}_{2}\right) F^{j_{3} 1}\left(\boldsymbol{p}_{3}\right)\right],
\end{array}
$$

and for the $\Delta$ baryon

$$
\begin{array}{r}
f_{\Delta}^{1 / 2} T_{\Delta}=-12 \sqrt{6} c_{0}^{\mathbf{1 0}, 1 / 2} \int\left[d k_{\perp}\right] T_{j_{1} j_{2} j_{3}, 1}^{111} F^{j_{1} 1}\left(\boldsymbol{p}_{1}\right) F^{j_{2} 1}\left(\boldsymbol{p}_{2}\right) F^{j_{3} 2}\left(\boldsymbol{p}_{3}\right), \\
f_{\Delta}^{1 / 2} V_{\Delta}=-12 \sqrt{6} c_{0}^{\mathbf{1 0}, 1 / 2} \int\left[d k_{\perp}\right] T_{j_{1} j_{2} j_{3}, 1}^{111}\left[F^{j_{1} 2}\left(\boldsymbol{p}_{1}\right) F^{j_{2} 1}\left(\boldsymbol{p}_{2}\right) F^{j_{3} 1}\left(\boldsymbol{p}_{3}\right)\right. \\
\left.+F^{j_{1} 1}\left(\boldsymbol{p}_{1}\right) F^{j_{2} 2}\left(\boldsymbol{p}_{2}\right) F^{j_{3} 1}\left(\boldsymbol{p}_{3}\right)\right], \\
f_{\Delta}^{1 / 2} A_{\Delta}=-12 \sqrt{6} c_{0}^{\mathbf{1 0}, 1 / 2} \int\left[d k_{\perp}\right] T_{j_{1} j_{2} j_{3}, 1}^{111}\left[F^{j_{1} 2}\left(\boldsymbol{p}_{1}\right) F^{j_{2} 1}\left(\boldsymbol{p}_{2}\right) F^{j_{3} 1}\left(\boldsymbol{p}_{3}\right)\right. \\
\left.-F^{j_{1} 1}\left(\boldsymbol{p}_{1}\right) F^{j_{2} 2}\left(\boldsymbol{p}_{2}\right) F^{j_{3} 1}\left(\boldsymbol{p}_{3}\right)\right],
\end{array}
$$

By summing over the isospin indices, we obtain the explicit expressions of the distribution amplitudes for the nucleon

$$
\begin{aligned}
f_{N} T_{N}= & 2 \sqrt{3} c_{0}^{\mathbf{8}, 1 / 2} \int\left[d k_{\perp}\right]\left[2 f_{\|}\left(\boldsymbol{k}_{\mathbf{1}}\right) f_{\|}\left(\boldsymbol{k}_{\mathbf{2}}\right) f_{\|}\left(\boldsymbol{k}_{\mathbf{3}}\right)\right. \\
& \left.+k_{1 L} k_{3 R} f_{\perp}\left(\boldsymbol{k}_{\mathbf{1}}\right) f_{\perp}\left(\boldsymbol{k}_{\mathbf{3}}\right) f_{\|}\left(\boldsymbol{k}_{\mathbf{2}}\right)+k_{2 L} k_{3 R} f_{\perp}\left(\boldsymbol{k}_{\mathbf{2}}\right) f_{\perp}\left(\boldsymbol{k}_{\mathbf{3}}\right) f_{\|}\left(\boldsymbol{k}_{\mathbf{1}}\right)\right] \\
f_{N} V_{N}= & 2 \sqrt{3} c_{0}^{\mathbf{8}, 1 / 2} \int\left[d k_{\perp}\right]\left[2 f_{\|}\left(\boldsymbol{k}_{\mathbf{1}}\right) f_{\|}\left(\boldsymbol{k}_{\mathbf{2}}\right) f_{\|}\left(\boldsymbol{k}_{\mathbf{3}}\right)\right. \\
& -k_{2 L} k_{1 R} f_{\perp}\left(\boldsymbol{k}_{\mathbf{2}}\right) f_{\perp}\left(\boldsymbol{k}_{\mathbf{1}}\right) f_{\|}\left(\boldsymbol{k}_{\mathbf{3}}\right)+k_{1 L} k_{2 R} f_{\perp}\left(\boldsymbol{k}_{\mathbf{1}}\right) f_{\perp}\left(\boldsymbol{k}_{\mathbf{2}}\right) f_{\|}\left(\boldsymbol{k}_{\mathbf{3}}\right) \\
& \left.+2 k_{3 L} k_{1 R} f_{\perp}\left(\boldsymbol{k}_{\mathbf{3}}\right) f_{\perp}\left(\boldsymbol{k}_{\mathbf{1}}\right) f_{\|}\left(\boldsymbol{k}_{\mathbf{2}}\right)+2 k_{3 L} k_{2 R} f_{\perp}\left(\boldsymbol{k}_{\mathbf{3}}\right) f_{\perp}\left(\boldsymbol{k}_{\mathbf{2}}\right) f_{\|}\left(\boldsymbol{k}_{\mathbf{1}}\right)\right], \\
f_{N} A_{N}= & 2 \sqrt{3} c_{0}^{\mathbf{8}, 1 / 2} \int\left[d k_{\perp}\right]\left[-k_{2 L} k_{1 R} f_{\perp}\left(\boldsymbol{k}_{\mathbf{2}}\right) f_{\perp}\left(\boldsymbol{k}_{\mathbf{1}}\right) f_{\|}\left(\boldsymbol{k}_{\mathbf{3}}\right)\right. \\
& +k_{1 L} k_{2 R} f_{\perp}\left(\boldsymbol{k}_{\mathbf{1}}\right) f_{\perp}\left(\boldsymbol{k}_{\mathbf{2}}\right) f_{\|}\left(\boldsymbol{k}_{\mathbf{3}}\right)+2 k_{3 L} k_{1 R} f_{\perp}\left(\boldsymbol{k}_{\mathbf{3}}\right) f_{\perp}\left(\boldsymbol{k}_{\mathbf{1}}\right) f_{\|}\left(\boldsymbol{k}_{\mathbf{2}}\right) \\
& \left.-2 k_{3 L} k_{2 R} f_{\perp}\left(\boldsymbol{k}_{\mathbf{3}}\right) f_{\perp}\left(\boldsymbol{k}_{\mathbf{2}}\right) f_{\|}\left(\boldsymbol{k}_{\mathbf{1}}\right)\right]
\end{aligned}
$$



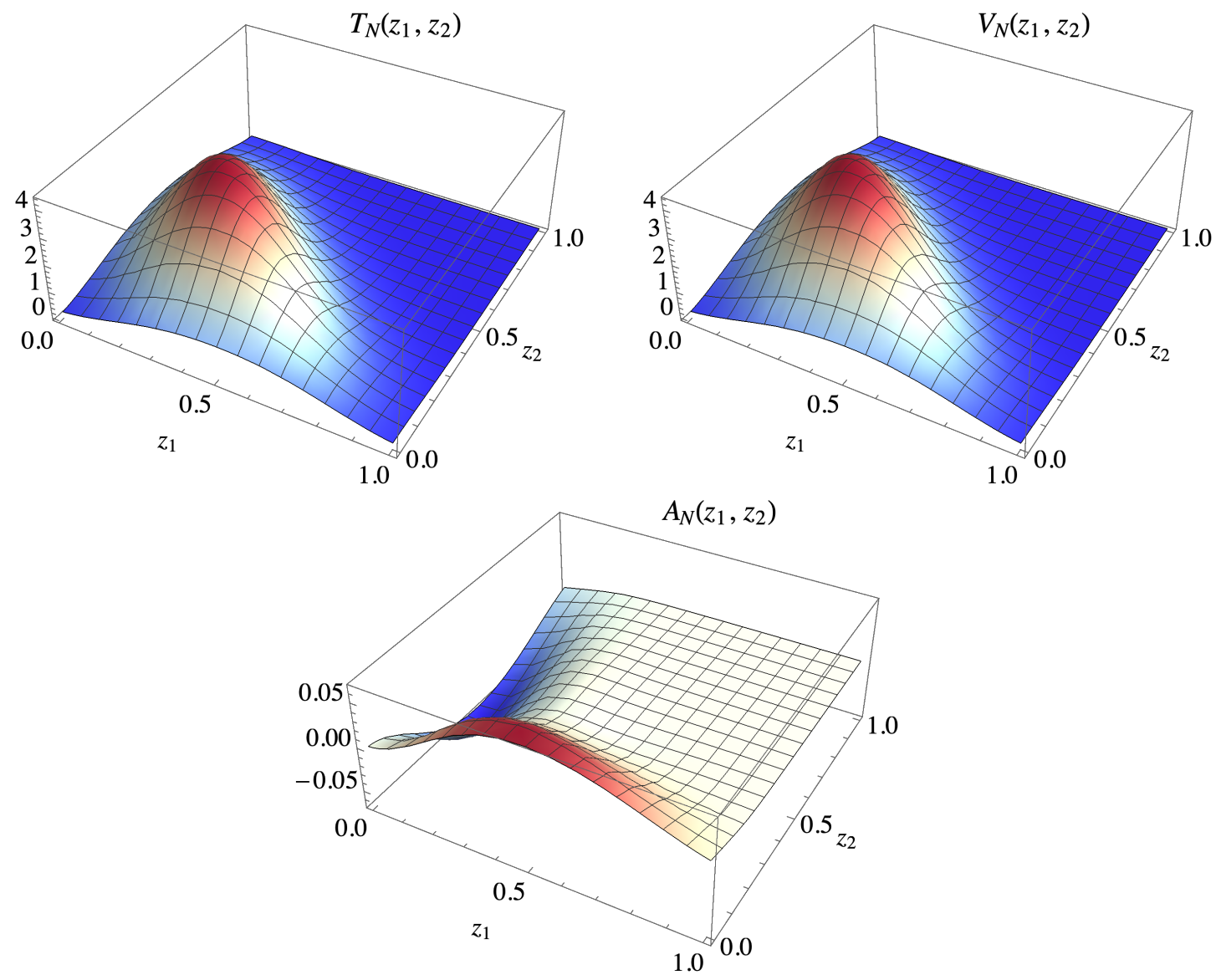

Figure 7. Nucleon distribution amplitudes $T_{N}, V_{N}$ and $A_{N}$.

and for the $\Delta$ baryon

$$
\begin{aligned}
f_{\Delta}^{1 / 2} T_{\Delta}= & -\frac{12}{\sqrt{5}} c_{0}^{\mathbf{1 0}, 1 / 2} \int\left[d k_{\perp}\right]\left[f_{\|}\left(\boldsymbol{k}_{1}\right) f_{\|}\left(\boldsymbol{k}_{2}\right) f_{\|}\left(\boldsymbol{k}_{3}\right)\right. \\
& \left.+k_{1 L} k_{3 R} f_{\perp}\left(\boldsymbol{k}_{1}\right) f_{\perp}\left(\boldsymbol{k}_{3}\right) f_{\|}\left(\boldsymbol{k}_{2}\right)+k_{2 L} k_{3 R} f_{\perp}\left(\boldsymbol{k}_{2}\right) f_{\perp}\left(\boldsymbol{k}_{3}\right) f_{\|}\left(\boldsymbol{k}_{1}\right)\right], \\
f_{\Delta}^{1 / 2} V_{\Delta}= & -\frac{12}{\sqrt{5}} c_{0}^{\mathbf{1 0}, 1 / 2} \int\left[d k_{\perp}\right]\left[2 f_{\|}\left(\boldsymbol{k}_{1}\right) f_{\|}\left(\boldsymbol{k}_{2}\right) f_{\|}\left(\boldsymbol{k}_{3}\right)\right. \\
& -k_{2 L} k_{1 R} f_{\perp}\left(\boldsymbol{k}_{2}\right) f_{\perp}\left(\boldsymbol{k}_{1}\right) f_{\|}\left(\boldsymbol{k}_{3}\right)-k_{1 L} k_{2 R} f_{\perp}\left(\boldsymbol{k}_{1}\right) f_{\perp}\left(\boldsymbol{k}_{2}\right) f_{\|}\left(\boldsymbol{k}_{3}\right) \\
& \left.-k_{3 L} k_{1 R} f_{\perp}\left(\boldsymbol{k}_{3}\right) f_{\perp}\left(\boldsymbol{k}_{1}\right) f_{\|}\left(\boldsymbol{k}_{2}\right)-k_{3 L} k_{2 R} f_{\perp}\left(\boldsymbol{k}_{3}\right) f_{\perp}\left(\boldsymbol{k}_{2}\right) f_{\|}\left(\boldsymbol{k}_{1}\right)\right], \\
f_{\Delta}^{1 / 2} A_{\Delta}= & \frac{12}{\sqrt{5}} c_{0}^{\mathbf{1 0}, 1 / 2} \int\left[d k_{\perp}\right]\left[k_{2 L} k_{1 R} f_{\perp}\left(\boldsymbol{k}_{2}\right) f_{\perp}\left(\boldsymbol{k}_{1}\right) f_{\|}\left(\boldsymbol{k}_{3}\right)\right. \\
& -k_{1 L} k_{2 R} f_{\perp}\left(\boldsymbol{k}_{1}\right) f_{\perp}\left(\boldsymbol{k}_{2}\right) f_{\|}\left(\boldsymbol{k}_{3}\right)+k_{3 L} k_{1 R} f_{\perp}\left(\boldsymbol{k}_{3}\right) f_{\perp}\left(\boldsymbol{k}_{1}\right) f_{\|}\left(\boldsymbol{k}_{2}\right) \\
& \left.-k_{3 L} k_{2 R} f_{\perp}\left(\boldsymbol{k}_{3}\right) f_{\perp}\left(\boldsymbol{k}_{2}\right) f_{\|}\left(\boldsymbol{k}_{1}\right)\right] .
\end{aligned}
$$



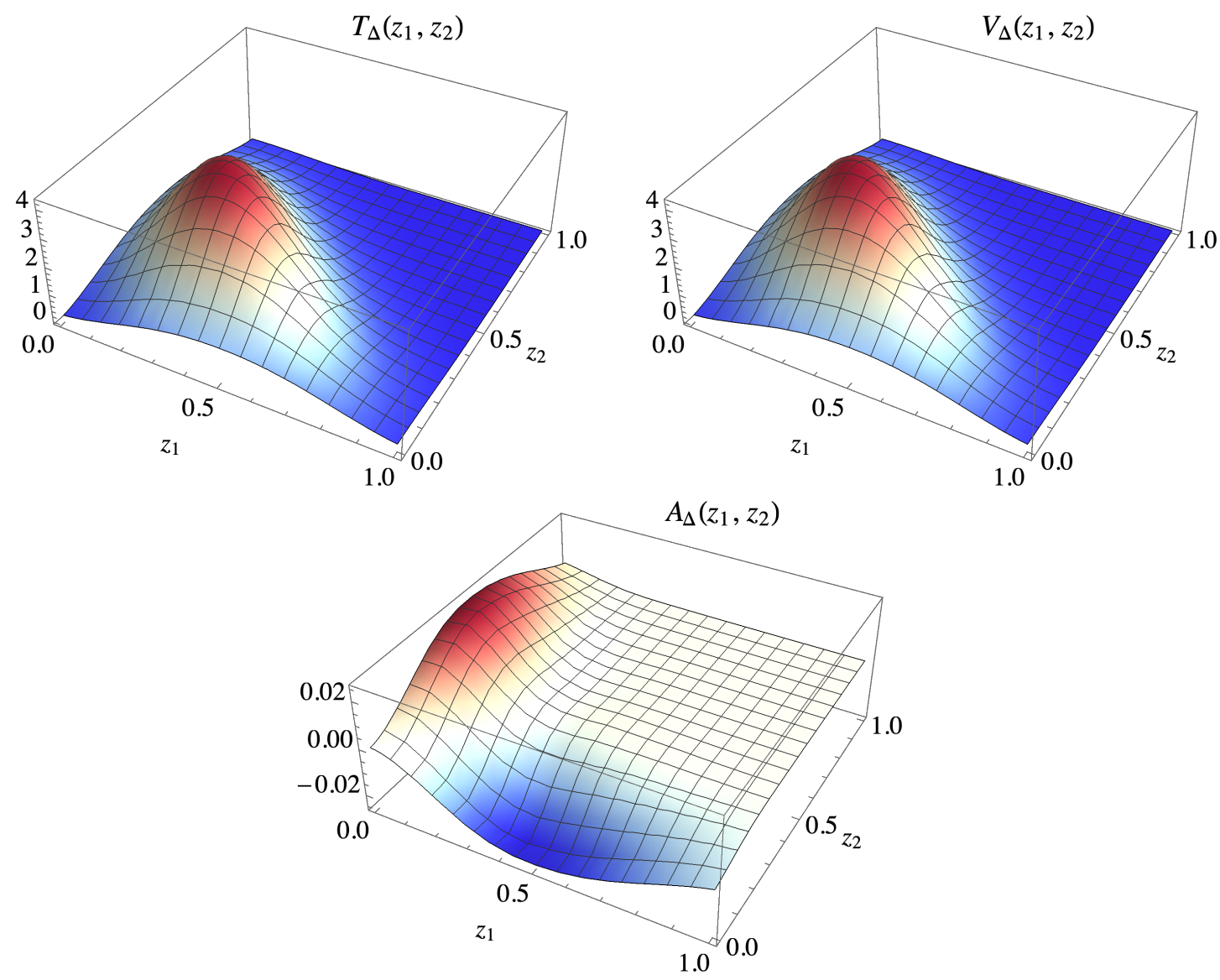

Figure 8. $\Delta$ baryon distribution amplitudes $T_{\Delta}, V_{\Delta}$ and $A_{\Delta}$.

In figure 7, the decomposed distribution amplitudes of the nucleon are shown. The antisymmetric distribution amplitude $A_{N}$ is numerically rather small compared with the symmetric ones $V_{N}$ and $T_{N}$.

In figure 8 , the decomposed distribution amplitudes of the $\Delta$ baryon are shown. The antisymmetric distribution amplitude $A_{\Delta}$ is numerically rather small compared with the symmetric ones $V_{\Delta}$ and $T_{\Delta}$.

Open Access. This article is distributed under the terms of the Creative Commons Attribution License (CC-BY 4.0), which permits any use, distribution and reproduction in any medium, provided the original author(s) and source are credited.

\section{References}

[1] S.J. Brodsky, H.-C. Pauli and S.S. Pinsky, Quantum chromodynamics and other field theories on the light cone, Phys. Rept. 301 (1998) 299 [hep-ph/9705477] [INSPIRE].

[2] G.P. Lepage and S.J. Brodsky, Exclusive processes in perturbative quantum chromodynamics, Phys. Rev. D 22 (1980) 2157 [InSPIRE]. 
[3] V.L. Chernyak and A.R. Zhitnitsky, Asymptotic behavior of exclusive processes in QCD, Phys. Rept. 112 (1984) 173 [INSPIRE].

[4] Z. Dziembowski, On uniqueness of relativistic nucleon state, Phys. Rev. D 37 (1988) 768 [INSPIRE].

[5] X.-D. Ji, J.-P. Ma and F. Yuan, Three quark light cone amplitudes of the proton and quark orbital motion dependent observables, Nucl. Phys. B 652 (2003) 383 [hep-ph/0210430] [INSPIRE].

[6] X.-D. Ji, J.-P. Ma and F. Yuan, Classification and asymptotic scaling of hadrons' light cone wave function amplitudes, Eur. Phys. J. C 33 (2004) 75 [hep-ph/0304107] [InSPIRE].

[7] S. Boffi, B. Pasquini and M. Traini, Linking generalized parton distributions to constituent quark models, Nucl. Phys. B 649 (2003) 243 [hep-ph/0207340] [INSPIRE].

[8] S. Boffi, B. Pasquini and M. Traini, Helicity dependent generalized parton distributions in constituent quark models, Nucl. Phys. B 680 (2004) 147 [hep-ph/0311016] [InSPIRE].

[9] B. Pasquini, M. Pincetti and S. Boffi, Chiral-odd generalized parton distributions in constituent quark models, Phys. Rev. D 72 (2005) 094029 [hep-ph/0510376] [InSPIRE].

[10] B. Pasquini and S. Boffi, Nucleon spin densities in a light-front constituent quark model, Phys. Lett. B 653 (2007) 23 [arXiv:0705.4345] [INSPIRE].

[11] C. Lorce, B. Pasquini and M. Vanderhaeghen, Unified framework for generalized and transverse-momentum dependent parton distributions within a $3 Q$ light-cone picture of the nucleon, JHEP 05 (2011) 041 [arXiv: 1102.4704] [INSPIRE].

[12] J. Bolz and P. Kroll, Modeling the nucleon wave function from soft and hard processes, Z. Phys. A 356 (1996) 327 [hep-ph/9603289] [INSPIRE].

[13] M. Diehl, T. Feldmann, R. Jakob and P. Kroll, Linking parton distributions to form-factors and Compton scattering, Eur. Phys. J. C 8 (1999) 409 [hep-ph/9811253] [INSPIRE].

[14] V.M. Braun, T. Lautenschlager, A.N. Manashov and B. Pirnay, Higher twist parton distributions from light-cone wave functions, Phys. Rev. D 83 (2011) 094023 [arXiv:1103.1269] [INSPIRE].

[15] B. Pasquini and S. Rodini, The twist-three distribution $e^{q}\left(x, k_{\perp}\right)$ in a light-front model, Phys. Lett. B 788 (2019) 414 [arXiv:1806.10932] [inSPIRE].

[16] B. Pasquini, S. Cazzaniga and S. Boffi, Transverse momentum dependent parton distributions in a light-cone quark model, Phys. Rev. D 78 (2008) 034025 [arXiv:0806.2298] [INSPIRE].

[17] G.P. Lepage and S.J. Brodsky, Exclusive processes in quantum chromodynamics: evolution equations for hadronic wave functions and the form-factors of mesons, Phys. Lett. B 87 (1979) 359 [INSPIRE].

[18] A.V. Efremov and A.V. Radyushkin, Factorization and asymptotical behavior of pion form-factor in QCD, Phys. Lett. B 94 (1980) 245 [INSPIRE].

[19] G.R. Farrar and D.R. Jackson, The pion form-factor, Phys. Rev. Lett. 43 (1979) 246 [INSPIRE].

[20] A. Duncan and A.H. Mueller, Asymptotic behavior of composite particle form-factors and the renormalization group, Phys. Rev. D 21 (1980) 1636 [InSPIRE]. 
[21] S.J. Brodsky, G.P. Lepage and S.A.A. Zaidi, Weak and electromagnetic form-factors of baryons at large momentum transfer, Phys. Rev. D 23 (1981) 1152 [InSPIRE].

[22] V.L. Chernyak and I.R. Zhitnitsky, Nucleon wave function and nucleon form-factors in QCD, Nucl. Phys. B 246 (1984) 52 [InSPIRE].

[23] M. Gari and N.G. Stefanis, Quark distribution amplitudes for the nucleon from perturbative QCD and QCD sum rules, Phys. Rev. D 35 (1987) 1074 [inSPIRE].

[24] I.D. King and C.T. Sachrajda, Nucleon wave functions and QCD sum rules, Nucl. Phys. B 279 (1987) 785 [INSPIRE].

[25] C.E. Carlson, M. Gari and N.G. Stefanis, Testing nucleon distribution amplitudes: relations between neutron and NA form-factors, Phys. Rev. Lett. 58 (1987) 1308 [INSPIRE].

[26] V.L. Chernyak, A.A. Ogloblin and I.R. Zhitnitsky, On the nucleon wave function, Sov. J. Nucl. Phys. 48 (1988) 536 [Yad. Fiz. 48 (1988) 841] [InSPIRE].

[27] V.L. Chernyak, A.A. Ogloblin and I.R. Zhitnitsky, Wave functions of octet baryons, Z. Phys. C 42 (1989) 569 [Yad. Fiz. 48 (1988) 1410] [Sov. J. Nucl. Phys. 48 (1988) 896] [InSPIRE].

[28] M. Bergmann and N.G. Stefanis, Bounds on $\frac{\left.\mid G_{M}^{n}\right) \mid}{G_{M}^{p}}$ from QCD sum rules, Phys. Rev. D 48 (1993) R2990 [INSPIRE].

[29] V.M. Braun, A. Lenz, N. Mahnke and E. Stein, Light cone sum rules for the nucleon form-factors, Phys. Rev. D 65 (2002) 074011 [hep-ph/0112085] [INSPIRE].

[30] N.G. Stefanis, The physics of exclusive reactions in QCD: theory and phenomenology, Eur. Phys. J. direct 1 (1999) 7 [hep-ph/9911375] [INSPIRE].

[31] P. Wein and A. Schäfer, Model-independent calculation of $\mathrm{SU}(3)_{f}$ violation in baryon octet light-cone distribution amplitudes, JHEP 05 (2015) 073 [arXiv:1501.07218] [INSPIRE].

[32] Y.-L. Liu and M.-Q. Huang, Distribution amplitudes of $\Sigma$ and $\Lambda$ and their electromagnetic form factors, Nucl. Phys. A 821 (2009) 80 [arXiv:0811.1812] [INSPIRE].

[33] Y.-L. Liu, M.-Q. Huang and D.-W. Wang, Light-cone QCD sum rules for $\Lambda$ baryon electromagnetic form factors and its magnetic moment, Eur. Phys. J. C 60 (2009) 593 [arXiv:0810.4973] [INSPIRE].

[34] Y.-L. Liu and M.-Q. Huang, Light-cone distribution amplitudes of $\Xi$ and their applications, Phys. Rev. D 80 (2009) 055015 [arXiv:0909.0372] [INSPIRE].

[35] G.R. Farrar, H. Zhang, A.A. Ogloblin and I.R. Zhitnitsky, Baryon wave functions and cross-sections for photon annihilation to baryon pairs, Nucl. Phys. B 311 (1989) 585 [INSPIRE].

[36] V.M. Braun, S.E. Derkachov, G.P. Korchemsky and A.N. Manashov, Baryon distribution amplitudes in QCD, Nucl. Phys. B 553 (1999) 355 [hep-ph/9902375] [INSPIRE].

[37] B. Pire, K. Semenov-Tian-Shansky and L. Szymanowski, $\pi N$ transition distribution amplitudes: their symmetries and constraints from chiral dynamics, Phys. Rev. D 84 (2011) 074014 [arXiv: 1106.1851$]$ [INSPIRE].

[38] B. Pire, K. Semenov-Tian-Shansky and L. Szymanowski, Transition distribution amplitudes and hard exclusive reactions with baryon number transfer, Phys. Rept. 940 (2021) 2185 [arXiv:2103.01079] [INSPIRE]. 
[39] V. Braun, R.J. Fries, N. Mahnke and E. Stein, Higher twist distribution amplitudes of the nucleon in QCD, Nucl. Phys. B 589 (2000) 381 [Erratum ibid. 607 (2001) 433] [hep-ph/0007279] [INSPIRE].

[40] V.M. Braun, A. Lenz and M. Wittmann, Nucleon form factors in QCD, Phys. Rev. D 73 (2006) 094019 [hep-ph/0604050] [INSPIRE].

[41] T.M. Aliev, K. Azizi and M. Savci, Electromagnetic form factors of octet baryons in QCD, Phys. Lett. B $\mathbf{7 2 3}$ (2013) 145 [arXiv: 1303.6798] [InSPIRE].

[42] I.V. Anikin, V.M. Braun and N. Offen, Nucleon form factors and distribution amplitudes in QCD, Phys. Rev. D 88 (2013) 114021 [arXiv:1310.1375] [INSPIRE].

[43] QCDSF collaboration, Nucleon distribution amplitudes and proton decay matrix elements on the lattice, Phys. Rev. D 79 (2009) 034504 [arXiv:0811.2712] [INSPIRE].

[44] QCDSF and UKQCD collaborations, Non-perturbative renormalization of three-quark operators, Nucl. Phys. B 812 (2009) 205 [arXiv:0810.3762] [InSPIRE].

[45] M. Gockeler et al., Nucleon distribution amplitudes from lattice QCD, Phys. Rev. Lett. 101 (2008) 112002 [arXiv:0804.1877] [INSPIRE].

[46] V.M. Braun et al., Light-cone distribution amplitudes of the nucleon and negative parity nucleon resonances from lattice QCD, Phys. Rev. D 89 (2014) 094511 [arXiv:1403.4189] [INSPIRE].

[47] G.S. Bali et al., Light-cone distribution amplitudes of the baryon octet, JHEP 02 (2016) 070 [arXiv: 1512.02050] [INSPIRE].

[48] RQCD collaboration, Light-cone distribution amplitudes of octet baryons from lattice QCD, Eur. Phys. J. A 55 (2019) 116 [arXiv:1903.12590] [InSPIRE].

[49] C. Mezrag, J. Segovia, L. Chang and C.D. Roberts, Parton distribution amplitudes: revealing correlations within the proton and Roper, Phys. Lett. B 783 (2018) 263 [arXiv: 1711.09101] [INSPIRE].

[50] C. Mezrag, J. Segovia, M. Ding, L. Chang and C.D. Roberts, Nucleon parton distribution amplitude: a scalar diquark picture, Springer Proc. Phys. 238 (2020) 773 [arXiv: 1811.09104] [INSPIRE].

[51] Z. Dziembowski, Nucleon distribution amplitudes from a relativistic quark model, Phys. Rev. D 37 (1988) 2030 [INSPIRE].

[52] N.G. Stefanis and M. Bergmann, Analysis of $\Delta^{+}(1232)$ isobar observables with improved quark distribution amplitudes, Phys. Lett. B 304 (1993) 24 [hep-ph/9211251] [INSPIRE].

[53] N.G. Stefanis and M. Bergmann, On the nucleon distribution amplitude: the heterotic solution, Phys. Rev. D 47 (1993) R3685 [hep-ph/9211250] [INSPIRE].

[54] M. Bergmann and N.G. Stefanis, Heterotic approach to the nucleon distribution amplitude, Phys. Lett. B 325 (1994) 183 [hep-ph/9403209] [INSPIRE].

[55] J. Bolz, R. Jakob, P. Kroll, M. Bergmann and N.G. Stefanis, Neutron form-factor: Sudakov suppression and intrinsic transverse size effect, Phys. Lett. B 342 (1995) 345 [hep-ph/9407250] [INSPIRE].

[56] S.M.H. Wong, Color octet contribution in exclusive $p$ wave charmonium decay into octet and decuplet baryons, Eur. Phys. J. C 14 (2000) 643 [hep-ph/9903236] [InSPIRE]. 
[57] B. Pasquini, M. Pincetti and S. Boffi, Parton content of the nucleon from distribution amplitudes and transition distribution amplitudes, Phys. Rev. D 80 (2009) 014017 [arXiv:0905.4018] [INSPIRE].

[58] E. Witten, Baryons in the 1/n expansion, Nucl. Phys. B 160 (1979) 57 [InSPIRE].

[59] E. Witten, Global aspects of current algebra, Nucl. Phys. B 223 (1983) 422 [InSPIRE].

[60] D. Diakonov, V.Y. Petrov and P.V. Pobylitsa, A chiral theory of nucleons, Nucl. Phys. B 306 (1988) 809 [INSPIRE].

[61] M. Wakamatsu and H. Yoshiki, A chiral quark model of the nucleon, Nucl. Phys. A 524 (1991) 561 [INSPIRE].

[62] C.V. Christov et al., Baryons as nontopological chiral solitons, Prog. Part. Nucl. Phys. 37 (1996) 91 [hep-ph/9604441] [INSPIRE].

[63] D. Diakonov and V.Y. Petrov, Instanton based vacuum from Feynman variational principle, Nucl. Phys. B 245 (1984) 259 [InSPIRE].

[64] D. Diakonov, Instantons at work, Prog. Part. Nucl. Phys. 51 (2003) 173 [hep-ph/0212026] [INSPIRE].

[65] K. Goeke et al., Nucleon form-factors of the energy momentum tensor in the chiral quark-soliton model, Phys. Rev. D 75 (2007) 094021 [hep-ph/0702030] [INSPIRE].

[66] D. Diakonov, V. Petrov, P. Pobylitsa, M.V. Polyakov and C. Weiss, Nucleon parton distributions at low normalization point in the large $N_{c}$ limit, Nucl. Phys. B 480 (1996) 341 [hep-ph/9606314] [INSPIRE].

[67] D. Diakonov, V.Y. Petrov, P.V. Pobylitsa, M.V. Polyakov and C. Weiss, Unpolarized and polarized quark distributions in the large $N_{c}$ limit, Phys. Rev. D 56 (1997) 4069 [hep-ph/9703420] [INSPIRE].

[68] M. Wakamatsu and T. Kubota, Chiral symmetry and the nucleon structure functions, Phys. Rev. D 57 (1998) 5755 [hep-ph/9707500] [INSPIRE].

[69] M. Wakamatsu and T. Kubota, Chiral symmetry and the nucleon spin structure functions, Phys. Rev. D 60 (1999) 034020 [hep-ph/9809443] [INSPIRE].

[70] P. Schweitzer, D. Urbano, M.V. Polyakov, C. Weiss, P.V. Pobylitsa and K. Goeke, Transversity distributions in the nucleon in the large $N_{c}$ limit, Phys. Rev. D 64 (2001) 034013 [hep-ph/0101300] [INSPIRE].

[71] H.-D. Son, A. Tandogan and M.V. Polyakov, Nucleon quasi-parton distributions in the large $N_{c}$ limit, Phys. Lett. B 808 (2020) 135665 [arXiv:1911.01955] [INSPIRE].

[72] V.Y. Petrov, P.V. Pobylitsa, M.V. Polyakov, I. Bornig, K. Goeke and C. Weiss, Off-forward quark distributions of the nucleon in the large $N_{c}$ limit, Phys. Rev. D 57 (1998) 4325 [hep-ph/9710270] [INSPIRE].

[73] K. Goeke, M.V. Polyakov and M. Vanderhaeghen, Hard exclusive reactions and the structure of hadrons, Prog. Part. Nucl. Phys. 47 (2001) 401 [hep-ph/0106012] [INSPIRE].

[74] V.Y. Petrov and M.V. Polyakov, Light cone nucleon wave function in the quark soliton model, hep-ph/0307077 [INSPIRE].

[75] P.V. Pobylitsa, Baryon wave function in large- $N_{c}$ QCD: universality, nonlinear evolution equation and asymptotic limit, Phys. Rev. D 71 (2005) 076002 [hep-th/0412291] [INSPIRE]. 
[76] P.V. Pobylitsa, Baryon distribution amplitude: large- $N_{c}$ factorization, spin-flavor symmetry and soft-pion theorem, Phys. Rev. D 72 (2005) 016001 [hep-ph/0501159] [INSPIRE].

[77] D. Diakonov and V. Petrov, Baryons as Fock states of 3,5,.. quarks, Annalen Phys. 13 (2004) 637 [hep-ph/0409362] [INSPIRE].

[78] D. Diakonov and V. Petrov, Estimate of the $\Theta^{+}$width in the relativistic mean field approximation, Phys. Rev. D 72 (2005) 074009 [hep-ph/0505201] [INSPIRE].

[79] C. Lorce, Improvement of the $\Theta^{+}$width estimation method on the light cone, Phys. Rev. D 74 (2006) 054019 [hep-ph/0603231] [INSPIRE].

[80] C. Lorce, Tensor charges of light baryons in the infinite momentum frame, Phys. Rev. D $\mathbf{7 9}$ (2009) 074027 [arXiv:0708.4168] [INSPIRE].

[81] C. Lorce, Baryon vector and axial content up to the $7 Q$ component, Phys. Rev. D 78 (2008) 034001 [arXiv: 0708.3139] [INSPIRE].

[82] C. Lorce and B. Pasquini, Quark Wigner distributions and orbital angular momentum, Phys. Rev. D 84 (2011) 014015 [arXiv:1106.0139] [InSPIRE].

[83] C. Lorce, B. Pasquini, X. Xiong and F. Yuan, The quark orbital angular momentum from Wigner distributions and light-cone wave functions, Phys. Rev. D 85 (2012) 114006 [arXiv:1111.4827] [INSPIRE].

[84] D. Diakonov and V.Y. Petrov, A theory of light quarks in the instanton vacuum, Nucl. Phys. B 272 (1986) 457 [INSPIRE].

[85] D. Diakonov, M.V. Polyakov and C. Weiss, Hadronic matrix elements of gluon operators in the instanton vacuum, Nucl. Phys. B 461 (1996) 539 [hep-ph/9510232] [INSPIRE].

[86] W. Pauli and S.M. Dancoff, The pseudoscalar meson field with strong coupling, Phys. Rev. 62 (1942) 85 [INSPIRE].

[87] D. Diakonov, V.Y. Petrov and M. Praszalowicz, Nucleon mass and nucleon $\Sigma$ term, Nucl. Phys. B 323 (1989) 53 [INSPIRE].

[88] G.S. Danilov, I.T. Dyatlov and V.Y. Petrov, Evolution operator and quark structure of states in two-dimensional massless electrodynamics, Nucl. Phys. B 174 (1980) 68 [INSPIRE].

[89] A. Blotz, D. Diakonov, K. Goeke, N.W. Park, V. Petrov and P.V. Pobylitsa, The SU(3) Nambu-Jona-Lasinio soliton in the collective quantization formulation, Nucl. Phys. A $\mathbf{5 5 5}$ (1993) 765 [INSPIRE].

[90] C. Lorce, Exotic and non-exotic baryon properties on the light cone, Ph.D. thesis, Liege U., Liege, Belgium (2007) [arXiv:1010.1685] [INSPIRE].

[91] H.-C. Kim, M.V. Polyakov and K. Goeke, Nucleon tensor charges in the SU(2) chiral quark-soliton model, Phys. Rev. D 53 (1996) 4715 [hep-ph/9509283] [INSPIRE].

[92] M.V. Polyakov and H.-D. Son, Second Gegenbauer moment of a $\rho$-meson distribution amplitude, Phys. Rev. D 102 (2020) 114005 [arXiv: 2008.06270] [INSPIRE].

[93] M. Bergmann, W. Schroers and N.G. Stefanis, Large order trend of the anomalous dimensions spectrum of trilinear twist-three quark operators, Phys. Lett. B 458 (1999) 109 [hep-ph/9903339] [INSPIRE].

[94] N.G. Stefanis, A modern view on exclusive reactions: selected topics at the interface between perturbative and nonperturbative QCD, Acta Phys. Polon. B 25 (1994) 1777 [InSPIRE]. 
[95] M.V. Polyakov and P. Schweitzer, Forces inside hadrons: pressure, surface tension, mechanical radius, and all that, Int. J. Mod. Phys. A 33 (2018) 1830025

[arXiv: 1805.06596] [INSPIRE].

[96] J.-Y. Kim and H.-C. Kim, Energy-momentum tensor of the nucleon on the light front: Abel tomography case, Phys. Rev. D 104 (2021) 074019 [arXiv:2105.10279] [INSPIRE].

[97] H. Alharazin, D. Djukanovic, J. Gegelia and M.V. Polyakov, Chiral theory of nucleons and pions in the presence of an external gravitational field, Phys. Rev. D 102 (2020) 076023 [arXiv: 2006. 05890] [INSPIRE].

[98] J.Y. Panteleeva and M.V. Polyakov, Forces inside the nucleon on the light front from $3 D$ Breit frame force distributions: Abel tomography case, Phys. Rev. D 104 (2021) 014008 [arXiv: 2102.10902] [INSPIRE].

[99] J. Gegelia and M.V. Polyakov, A bound on the nucleon Druck-term from chiral EFT in curved space-time and mechanical stability conditions, Phys. Lett. B 820 (2021) 136572 [arXiv: 2104.13954] [INSPIRE].

[100] M.V. Polyakov and P. Schweitzer, Mechanical properties of particles, PoS SPIN2018 (2019) 066 [arXiv: 1812.06143] [InSPIRE].

[101] J.Y. Panteleeva and M.V. Polyakov, Quadrupole pressure and shear forces inside baryons in the large $N_{c}$ limit, Phys. Lett. B 809 (2020) 135707 [arXiv: 2004.02912] [INSPIRE].

[102] J.-Y. Kim and B.-D. Sun, Gravitational form factors of a baryon with spin-3/2, Eur. Phys. J. C 81 (2021) 85 [arXiv: 2011.00292] [InSPIRE].

[103] G.-S. Yang, H.-C. Kim, M.V. Polyakov and M. Praszałowicz, Pion mean fields and heavy baryons, Phys. Rev. D 94 (2016) 071502 [arXiv: 1607.07089] [InSPIRE].

[104] H.-C. Kim, M.V. Polyakov and M. Praszałowicz, Possibility of the existence of charmed exotica, Phys. Rev. D 96 (2017) 014009 [Addendum ibid. 96 (2017) 039902] [arXiv: 1704.04082] [INSPIRE].

[105] H.-C. Kim, M.V. Polyakov, M. Praszalowicz and G.-S. Yang, Strong decays of exotic and nonexotic heavy baryons in the chiral quark-soliton model, Phys. Rev. D 96 (2017) 094021 [Erratum ibid. 97 (2018) 039901] [arXiv:1709.04927] [INSPIRE].

[106] J.-Y. Kim, H.-C. Kim and G.-S. Yang, Mass spectra of singly heavy baryons in a self-consistent chiral quark-soliton model, Phys. Rev. D 98 (2018) 054004 [arXiv: 1801.09405] [INSPIRE].

[107] G.-S. Yang and H.-C. Kim, Magnetic moments of the lowest-lying singly heavy baryons, Phys. Lett. B 781 (2018) 601 [arXiv:1802.05416] [InSPIRE].

[108] J.-Y. Kim and H.-C. Kim, Electromagnetic form factors of singly heavy baryons in the self-consistent SU(3) chiral quark-soliton model, Phys. Rev. D 97 (2018) 114009 [arXiv: 1803.04069] [INSPIRE].

[109] H.-C. Kim, Heavy baryons in a pion mean-field approach: a brief review, J. Korean Phys. Soc. 73 (2018) 165 [arXiv:1804.04393] [INSPIRE].

[110] J.-Y. Kim and H.-C. Kim, Improved pion mean fields and masses of singly heavy baryons, PTEP 2020 (2020) 043D03 [arXiv: 1909.00123] [InSPIRE].

[111] G.-S. Yang and H.-C. Kim, Magnetic transitions and radiative decays of singly heavy baryons, Phys. Lett. B 801 (2020) 135142 [arXiv:1909.03156] [INSPIRE]. 
[112] J.-Y. Kim and H.-C. Kim, Pion mass dependence of the electromagnetic form factors of singly heavy baryons, PTEP 2021 (2021) 063D03 [arXiv: 1912.01437] [INSPIRE].

[113] G.-S. Yang and H.-C. Kim, Isospin mass differences of singly heavy baryons, Phys. Lett. B 808 (2020) 135619 [arXiv: 2004.08524] [INSPIRE].

[114] J.-Y. Kim, H.-C. Kim, M.V. Polyakov and H.-D. Son, Strong force fields and stabilities of the nucleon and singly heavy baryon $\Sigma_{c}$, Phys. Rev. D 103 (2021) 014015 [arXiv: 2008. 06652] [INSPIRE].

[115] J.-Y. Kim and H.-C. Kim, Electric quadrupole form factors of singly heavy baryons with spin 3/2, PTEP 2021 (2021) 023D02 [arXiv: 2008.08296] [INSPIRE].

[116] Fayyazuddin and Riazuddin, Modern introduction to particle physics, World Scientific, River Edge, NJ, U.S.A. (2012). 